\title{
Discrete-Time Mixed-Integer Programming Models and Solution Methods for Production Scheduling in Multistage Facilities
}

\author{
Andres F. Merchan, Hojae Lee, Christos T. Maravelias* \\ Department of Chemical and Biological Engineering, University of Wisconsin-Madison \\ 1415 Engineering Drive, Madison, WI 53706, USA
}

\begin{abstract}
We address the problem of production scheduling in multi-product multistage batch plants. Unlike most of the previous works, which propose continuoustime models, we study discrete-time mixed-integer programming models and solution methods. Specifically, we discuss two models based on network representations of the facility and develop two new models inspired by the Resource-Constrained Project Scheduling Problem. Furthermore, we propose different solution methods, including tightening methods based on processing unit availability, a reformulation based on processing unit occupancy, and an algorithm to refine approximate solutions for large-scale instances. Finally, we present a comprehensive computational study, which shows that speedups of up to four orders of magnitude are observed when our models and methods are compared to existing approaches.
\end{abstract}

Keywords: Sequential production environments, resource-constrained project scheduling, tightening constraints, reformulations.

\section{Introduction}

Production scheduling is one of the main planning functions within the scope of process operations. It can be defined as a decision-making process in which a set of limited shared resources is allocated to production tasks over time (Pinedo, 2012). Although originally regarded as a feasibility problem, production scheduling is often associated with the optimization of an objective that is concerned with the adequate use of installed resources, customer satisfaction, and/or operation costs. Scheduling encounters applications in a wide range of process industries, from large-scale oil operations, to medium-scale commodities production, to small-scale pharmaceuticals manufacturing. It has been the subject of active research within the Process Systems Engineering (PSE) community for the last three decades (Floudas and Lin, 2004; Harjunkoski et al., 2014; Kallrath, 2002; Maravelias, 2012; Mendez et al., 2006; Pinto and Grossmann, 1998; Reklaitis, 1996). While a range of exact and approximate solution approaches have been studied, exact methods based on mathematical programming have been the most popular approach.

Early works followed the related discrete manufacturing literature, by considering facilities that resembled the structure of jobs and machines in series or in parallel studied in the manufacturing sector (Egli and Rippin, 1986; Mauderli and Rippin, 1979). Later on, Pantelides and coworkers (Kondili et al., 1993; Pantelides, 1994; Schilling and Pantelides, 1996; Shah et al., 1993) proposed different frameworks and models to address problems in facilities with complex topology and various types of resources as commonly encountered in the chemical process industry. Since then, chemical facilities have been broadly classified according to process structure and restrictions on material handling into sequential and network environments (Maravelias, 2012; Mendez et al., 2006; Pinto and Grossmann, 1998). The former are characterized by the flow of batches throughout well-defined stages: every batch has to be trackable and follow a specific route; mixing and splitting are not allowed. The latter allow for more complex flow patterns, in which stages cannot be defined, batch mixing and splitting are permitted, and tasks can consume and produce multiple materials. 
Several models have been proposed for both environments in order to address common features such as changeovers, inventory cost and constraints, and utility requirements, among others. Batchbased models have been developed for problems in sequential environments while material-based models are used for network environments (Maravelias, 2012). Some recent works introduced the concept of hybrid environments to model processes that include both sequential and network subsystems (Sundaramoorthy and Maravelias, 2011; Velez and Maravelias, 2013).

A critical aspect of a mathematical model for scheduling is the time representation. On one hand, discrete-time models use a predefined grid onto which tasks, inventory levels, and other elements are mapped. On the other hand, continuous-time models use a variable grid that requires the location of time points to be included as a decision variable in the optimization model. Most of the work in the late 1990s and during the 2000s focused on developing continuous-time models for both sequential and network environments. Finally, models based on a mixed-time representation have also been proposed (Maravelias, 2005).

While continuous-time models are substantially smaller than their discrete-time counterparts, the latter have a number of advantages. They are conceptually simpler and thus can be readily modified to account for features such as setups and maintenance operations (Kondili et al., 1993); allow linear modeling of inventory and utility costs; and they can be easily extended to account for a variety of processing features (e.g., utilities, time-varying parameters, complex logistics) (Kelly and Zyngier, 2009; Velez and Maravelias, 2013; Velez and Maravelias, 2014). Furthermore, a recent computational study showed that discrete-time models are more effective not only in reducing computational time, but also in finding better solutions (Sundaramoorthy and Maravelias, 2011; Velez et al., 2015). Motivated by these advantages, researchers have looked into developing advanced solution methods for discrete-time MIP scheduling models for network production environments (Velez and Maravelias, 2014). The overwhelming majority of models for sequential environments, however, employ continuous representation of time.

The goal of this paper is twofold. First, we propose new discrete-time MIP models for the scheduling of multi-stage facilities, the most common sequential environment. Second, based upon ideas that have been used to enhance the solution of scheduling problems in network environments, we develop solution methods for the proposed discrete-time models.

The rest of the article is structured as follows. In section 2 , we formally define the problem under study. In section 3, we provide an extensive review of the relevant literature and concepts upon which our models and methods are based. In section 4, we define four discrete-time models for scheduling in sequential environments. In section 5 , several solution methods are proposed. Section 6 presents small-scale examples that include utility constraints. Section 7 contains an extensive computational study to test the proposed models and solution methods. We use uppercase italic letters for variables, uppercase bold letters for sets, lowercase italic letters for indices, and lowercase Greek letters for parameters.

\section{Problem statement}

We study the problem of short-term scheduling in multistage batch chemical plants, where batches of different products are processed in a series of stages, each of which consists of one or more unrelated parallel units, and each unit belongs to a single stage. Batches should be processed as discrete entities so their identity is preserved, thus mixing of different batches and splitting of a single batch into smaller quantities are not allowed. All the products follow the same sequence throughout the stages, although some products might skip one or more stages. This basic problem is hereinafter referred to as the Multistage Batch Plant Scheduling Problem, MBPSP. In addition, we consider the case where limited utilities are required. 

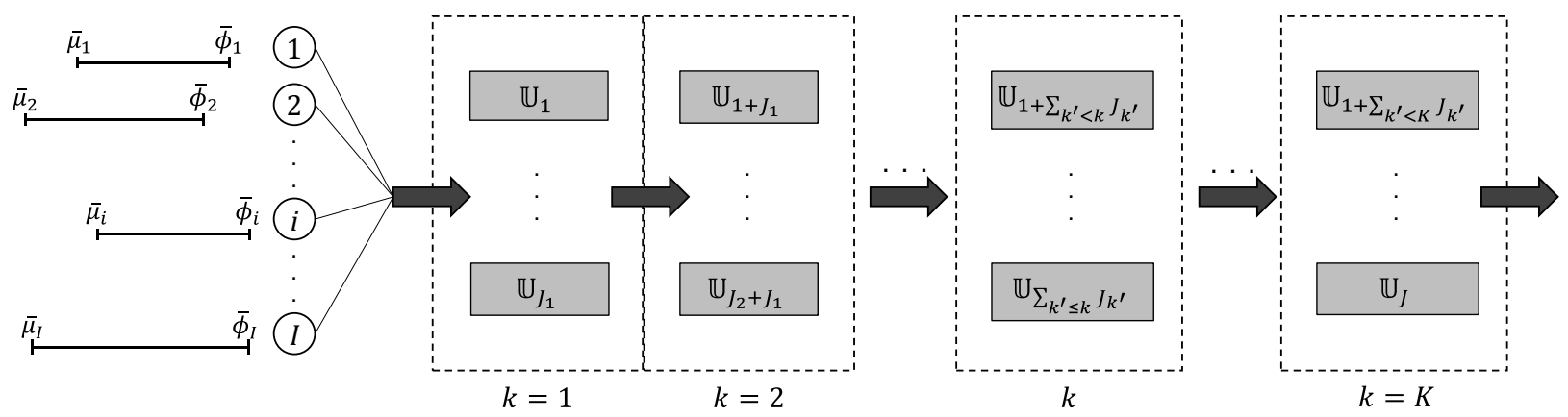

Figure 1. General multistage batch production facility.

Circles represent batches (time windows are given), gray boxes denote processing units, and dotted-line boxes define stages. $I \equiv|\mathbf{I}|$ is the total number of batches, $J \equiv|\mathbf{J}|$ denotes the total number of units, $K \equiv|\mathbf{K}|$ defines the number of stages, and $J_{k} \equiv\left|\mathbf{J}_{k}\right|$ is the total number of units on stage $k$.

We formally define the MBPSP by introducing the basic sets, indices and parameters that describe a multistage batch plant (see Figure 1). Extensions to include utilities are deferred to section 4.6.

Sets and indices

$i \in \mathbf{I}$

Batches

$j \in \mathbf{J}$

$k \in \mathbf{K}$

Processing units (denoted by $\mathbb{U}_{j}$ )

$\mathbf{I}_{j}$

Stages

$\mathbf{I}_{k}$

Batches that can be processed in unit $j \in \mathbf{J}$

Batches that are processed on stage $k \in \mathbf{K}$

$\mathbf{J}_{i}$

Units that can process batch $i \in \mathbf{I}$

$\mathbf{J}_{k}$

Units that belong to stage $k \in \mathbf{K}$

$\mathbf{J}_{i k}$

Units that can process batch $i \in \mathbf{I}$ on stage $k \in \mathbf{K}_{i}$, i.e. $\mathbf{J}_{i k} \equiv \mathbf{J}_{i} \cap \mathbf{J}_{k}$

$\mathbf{K}_{i}$

Stages on which batch $i \in \mathbf{I}$ is processed

Parameters

$\bar{\tau}_{i j}$

$\bar{\mu}_{i}$

$\bar{\phi}_{i}$

$\kappa_{i}^{F S} / \kappa_{i}^{L S}$

$v_{i k}$

Processing time of batch $i \in \mathbf{I}$ in unit $j \in \mathbf{J}_{i}$

Release time for batch $i \in \mathbf{I}$

Due date for batch $i \in \mathbf{I}$

First/last stage on which batch $i \in \mathbf{I}$ is processed

The next stage on which batch $i \in \mathbf{I}$ is processed after stage $k \in \mathbf{K}_{i}$, i.e. batch $i$ is processed on stage $k+v_{i k}$ immediately after stage $k$

$\alpha_{i j}$

Fixed cost of processing batch $i \in \mathbf{I}$ in $j \in \mathbf{J}_{i}$

The short-term scheduling problem of a multistage batch facility requires that every batch $i \in \mathbf{I}$ is processed exactly once on every stage $k \in \mathbf{K}_{i}$, following the route $1 \rightarrow 2 \rightarrow \cdots \rightarrow|\mathbf{K}|$, within its time window denoted by $\left[\bar{\mu}_{i}, \bar{\phi}_{i}\right]$. A unit can only process one batch at any given time, and once a batch $i \in \mathbf{I}$ starts being processed in unit $j \in \mathbf{J}_{i k}$ it cannot be stopped until it is completed, i.e. preemption is not allowed. Every unit belongs to a single stage, which means that $\left\{\mathbf{J}_{k}\right\}_{k \in \mathbf{K}}$ is a partition of the set J (i.e. $\mathbf{J}_{k} \neq \emptyset \forall k, \mathbf{J}=\bigcup_{k \in \mathbf{K}} \mathbf{J}_{k}$ and $\mathbf{J}_{k} \cap \mathbf{J}_{k^{\prime}}=\emptyset \forall k \neq k^{\prime}$ ). We further assume that there is unlimited intermediate storage (UIS) between stages. We do not consider switchover times. Three minimization objectives are considered: makespan, earliness and cost.

We identify three major types of constraints that must be satisfied: (i) the routing of a batch through different stages, henceforth referred to as batch precedence, (ii) unique assignment of a batch to every stage in which it is processed, hereinafter called batch-stage assignment, and (iii) unit utilization and sequencing of batches assigned to the same unit, henceforth referred to as unit assignment. The requirement that every batch has to be processed between its release and due dates can explicitly or implicitly be enforced through additional constraints or sets of time points. 


\section{Background}

\subsection{Modeling in sequential environments}

The first efforts to develop systematic methods for scheduling chemical production in sequential environments followed discrete manufacturing approaches, where the production of independent and enumerable items (e.g. vehicles, mechanical parts, furniture, electronics, etc.) has to be planned. A vast literature in the operations research (OR) community is dedicated to exact, heuristic and metaheuristic methods for discrete manufacturing scheduling (Graves, 1981; Maccarthy and Liu, 1993; Pinedo, 2005; Pinedo, 2012; Wiers, 1997). The problem we consider is similar to the Flexible (hybrid, compound, multiprocessor) Flow Shop (FFS) problem which has also been the subject of extensive research (Linn and Zhang, 1999; Ruiz and Vazquez-Rodriguez, 2010; Wang, 2005). However ${ }_{2}$ the MBPSP is more general since it considers features such as special storage policies for fluid materials and utility constraints.

The MBPSP has attracted the interest of several researchers in the PSE community (Floudas and Lin, 2004; Mendez et al., 2006; Pinto and Grossmann, 1998; Reklaitis, 1996; Shah, 1998). The vast majority of these works focus on developing continuous-time MIP models. Two major approaches have been adopted: (1) precedence-based models in which time is only a variable and sequencing is enforced through explicit binary variables (Gupta and Karimi, 2003; Harjunkoski and Grossmann, 2002; Kopanos et al., 2009; Mendez et al., 2000; Mendez et al., 2001), and (2) time-grid-based models in which time is both a variable and an index, and the sequencing is enforced by assigning one batch to a single time period with no extra variables required (Capon-Garcia et al., 2009; Castro and Grossmann, 2005; Chen et al., 2002; Liu and Karimi, 2007; Liu and Karimi, 2007; Pinto and Grossmann, 1995; Sundaramoorthy and Karimi, 2005). In turn, each approach can be subdivided in terms of global or local precedence for the former, and global or unit-specific time grid for the latter. The main advantage of the precedence-based approach is that no extra assumptions are required to model time. Its main disadvantage is that it can lead to relatively large model sizes, since the sequencing variables and constraints increase quadratically with the number of batches. On the other hand, the main benefit of the time-grid-based approach is a relatively small model size, but its main drawback is the iterative procedure it requires to determine the number of time points to include in the grid.

To reduce the difficulties in solving the MBPSP, various solution methods have been proposed. Pinto and Grossmann introduced preordering constraints and applied Benders decomposition to their slot-based model to solve large-scale instances (Pinto and Grossmann, 1995). Harjunkoski and Grossmann decomposed the problem into two levels: high-level MIP subproblem for the assignment decisions, and low-level constraint programming (CP) subproblem for sequencing (Harjunkoski and Grossmann, 2002). These subproblems are solved iteratively by adding integer cuts whenever infeasibilities occur. Another decomposition method also decomposes the problem into assignment and sequencing subproblems, but special-purpose sequencing algorithms enabled more efficient sequencing in the second level (Maravelias, 2006). Castro et al. introduced a decomposition algorithm for large-scale problems, in which only one or two orders are scheduled iteratively at a time, until the schedule of the complete set of orders is obtained (Castro et al., 2009). For the objective of makespan minimization specifically, Castro and Grossmann (Castro and Grossmann, 2005) extended the iterative approach originally proposed by Maravelias and Grossmann (Maravelias and Grossmann, 2003). In this approach, the initial time horizon of the problem is estimated and then increased by a time period in each iteration, until a feasible solution is obtained. 


\subsection{Continuous-time models in sequential environments}

In this work we choose a global precedence-based continuous-time model to compare against the discrete-time models we study in section 4 . The model we use was presented independently by Mendez et al. (Mendez et al., 2001) and Harjunkoski and Grossmann (Harjunkoski and Grossmann, 2002) as a generalization of the model first developed by Brah (Brah, 1988) for identical machines in each stage. It is one of the best continuous-time models for the MBPSP and has been used as a benchmark formulation in different studies (Castro and Grossmann, 2005; Castro et al., 2009; Harjunkoski and Grossmann, 2002). We refer to it as model MH\&C. It employs the following variables:

$C_{i k} \in \mathbb{R}_{+} \quad$ Completion time for batch $i \in \mathbf{I}$ on stage $k \in \mathbf{K}_{i}$

$X_{i j}^{C} \in\{0,1\} \quad$ One if batch $i \in \mathbf{I}$ is assigned to unit $j \in \mathbf{J}_{i}$

$Y_{i i^{\prime} k} \in\{0,1\} \quad$ One if batch $i \in \mathbf{I}$ is processed before batch $i^{\prime} \in \mathbf{I}$ on stage $k \in \mathbf{K}_{i} \cap \mathbf{K}_{i^{\prime}}$

Batch precedence is guaranteed using equation (1) that relates completion times of batch $i$ in consecutive stages. Batch-stage assignment is enforced through equation (2). Unit assignment and sequencing is modeled via equations (3) and (4) which guarantee proper sequencing of two batches assigned to the same unit. Finally, satisfaction of the time window is achieved through equations (5) and (6).

$$
\begin{aligned}
& C_{i\left(k+v_{i k}\right)} \geq C_{i k}+\sum_{j \in \mathbf{J}_{i\left(k+v_{i k}\right)}} \bar{\tau}_{i j} X_{i j}^{C} \quad \forall i \in \mathbf{I}, k \in \mathbf{K}_{i} \backslash\left\{\kappa_{i}^{L S}\right\} \\
& \sum_{j \in \mathbf{J}_{i k}} X_{i j}^{C}=1 \quad \forall i \in \mathbf{I}, k \in \mathbf{K}_{i} \\
& C_{i^{\prime} k}-\bar{\tau}_{i^{\prime} j} \geq C_{i k}-M\left[\left(1-Y_{i i^{\prime} k}\right)+\left(2-X_{i j}^{C}-X_{i^{\prime} j}^{C}\right)\right] \\
& C_{i k}-\bar{\tau}_{i j} \geq C_{i^{\prime} k}-M\left[Y_{i i^{\prime} k}+\left(2-X_{i j}^{C}-X_{i^{\prime} j}^{C}\right)\right] \\
& C_{i \kappa_{i}^{F S}} \geq \bar{\mu}_{i}+\sum_{j \in \mathbf{I}_{i K_{i}^{F S}}: i^{\prime}>i, k \in \mathbf{K}_{i} \cap \mathbf{K}_{i^{\prime}}, j \in \mathbf{J}_{i k} \cap \mathbf{J}_{i^{\prime} k}} \bar{\tau}_{i j} X_{i j}^{C} \quad \forall i \in \mathbf{I} \\
& C_{i \kappa_{i}^{L S}} \leq \bar{\phi}_{i} \quad \forall i \in \mathbf{I}
\end{aligned}
$$

For parameter $M$ in equations (3) and (4) we use the length of the scheduling horizon, i.e. $M=\max _{i \in \mathbf{I}} \bar{\phi}_{i}-\min _{i \in \mathbf{I}} \bar{\mu}_{i}$, although other values can be used without a significant change in computational performance. Finally, equations (7), (8), and (9) provide definitions for the minimization objectives of cost, earliness and makespan respectively. Equation (9) introduces an additional decision variable to represent the makespan, $M S \in \mathbb{R}_{+}$.

$$
\begin{aligned}
& \min \sum_{i \in \mathbf{I}, j \in \mathbf{J}_{i}} \alpha_{i j} X_{i j}^{C} \\
& \min \sum_{i \in \mathbf{I}}\left(\bar{\phi}_{i}-C_{i \kappa_{i}^{L S}}\right) \\
& \min M S: M S \geq C_{i \kappa_{i}^{L S}} \quad \forall i \in \mathbf{I}
\end{aligned}
$$


Although we mainly use model MH\&C to compare its performance versus the discrete-time models introduced in section 4, we also use it in section 5.5 as a tool to refine solutions obtained by discrete-time models.

\subsection{Discrete-time modeling in network environments}

So far we have focused on describing and modeling facilities in which the products follow welldefined routes through predetermined stages. These type of facilities are generalizations of the flow shop machine environment in discrete manufacturing. Most often, chemical production facilities handle fluids than can be mixed or split, and therefore it is common to relax the material handling restrictions. These types of facilities are referred to as network environments (Maravelias, 2012).

In terms of modeling, network environments are represented in terms of the tasks that define the production recipe. Two abstract representations have extensively been used in the PSE literature, the State-Task Network, STN (Kondili et al., 1993), and the Resource-Task Network, RTN (Pantelides, 1994). Although both the STN and RTN represent the facility as a network, the main difference between them is that the former only considers materials as resource nodes (units and other resources are implicitly mapped), while the latter includes processing units and other limited resources as nodes of the network. Both representations allow for either continuous or discrete modeling of time. In general, network processing relies on material balances to enforce sequencing constraints, and therefore their mathematical formulations are referred to as material-based models.

We provide discrete-time mathematical formulations for both representations below. For the discussion in the next two subsections we decouple tasks from processing units as follows: if a task can be performed in two units, then two new tasks are defined for the execution of the original task in each unit. We use superscript $N$ to indicate that a definition is applicable to both representations. Superscripts $S T N$ and $R T N$ are used whenever a definition is applicable to one representation.

\section{Sets and indices}

$i \in \mathbf{I}^{N} \quad$ Tasks

$r \in \mathbf{R}^{N} \quad$ General resources (materials, units, utilities, manpower, etc.)

$t \in \mathbf{T}^{N} \quad$ Time points, $\mathbf{T}^{N}=\left\{t \in \mathbb{Z}: 0 \leq t \leq \eta^{N}\right\}$

$\mathbf{I}_{r}^{N} \quad$ Tasks that consume or produce resource $r \in \mathbf{R}^{N}$

$\mathbf{I}_{r}^{+, N} / \mathbf{I}_{r}^{-, N} \quad$ Tasks that produce/consume resource $r \in \mathbf{R}^{N}$

$\mathbf{R}^{U, N} \quad$ Processing units

$\mathbf{R}_{i}^{N} / \mathbf{R}_{i}^{U, N} \quad$ General resources/units that are consumed or produced by task $i \in \mathbf{I}^{N}$

\section{Parameters}

$\beta_{i r}^{m, N} / \beta_{i r}^{M, N} \quad$ Minimum/maximum capacity utilization of resource $r \in \mathbf{R}^{N}$ by task $i \in \mathbf{I}_{r}^{N}$

$\delta^{N}$ Step size for time discretization

$\bar{\eta}^{N} / \eta^{N}$

$\xi_{r t}^{N}$

Scheduling horizon in real time units/in terms of time grid, $\eta^{N}=\left\lceil\bar{\eta}^{N} / \delta^{N}\right\rceil$

$\rho_{i r t}^{N}$ Amount of resource $r \in \mathbf{R}^{N}$ made available $\left(\xi_{r t}^{N}>0\right)$ or unavailable $\left(\xi_{r t}^{N}<0\right)$ at time $t \in \mathbf{T}^{N}$

$\bar{\tau}_{i}^{N} / \tau_{i}^{N}$

Number of variable units of resource $r \in \mathbf{R}^{N}$ that are required by task $i \in \mathbf{I}_{r}^{N} t$ time points after the beginning of the task

$\psi_{r t}^{N}$

Processing time of task $i \in \mathbf{I}^{N}$ in real time units/in terms of time grid, $\tau_{i}^{N}=\left\lceil\bar{\tau}_{i}^{N} / \delta^{N}\right\rceil$

Maximum storage allowed for resource $r \in \mathbf{R}^{N}$ during period $t \in \mathbf{T}^{N} \backslash\{0\}$

Decision variables

$X_{i t}^{N} \in\{0,1\} \quad$ It is equal to one if task $i \in \mathbf{I}^{N}$ starts at time $t \in \mathbf{T}^{N}$

$B_{i t}^{N} \geq 0 \quad$ Extent of task $i \in \mathbf{I}_{r}^{N}$ during period $t \in \mathbf{T}^{N} \backslash\{0\}$ 
$R_{r t}^{N} \geq 0 \quad$ Excess resource/available inventory of resource $r \in \mathbf{R}^{N}$ during period $t \in \mathbf{T}^{N} \backslash\{0\}$

\subsubsection{State-Task Network}

We consider a modification of the STN formulation (Shah et al., 1993), which henceforth is referred to as model SP\&S. Since, materials and units are treated differently in STN, let $\mathbf{R}^{M, S T N}$ represent materials and $\rho_{i r}^{S T N}=\rho_{i r t}^{N}$ be the conversion coefficient for task $i \in \mathbf{I}^{N}$ producing $\left(\rho_{i r}^{S T N}>0\right)$ or consuming $\left(\rho_{i r}^{S T N}<0\right)$ material $r \in \mathbf{R}^{M, S T N}$. Model SP\&S is defined through equations (10), (11), and (12). Equation (10) is a clique constraint that ensures a unit can only perform one task at a given time, equation (11) enforces material balances and storage capacities, and equation (12) guarantees that the batch sizes satisfy unit capacities.

$$
\begin{aligned}
& \sum_{i \in \mathbf{I}_{r}^{N}} \sum_{t^{\prime}=t-\tau_{i}^{N+1}}^{t} X_{i t^{\prime}}^{N} \leq 1 \quad \forall r \in \mathbf{R}^{U, N}, t \in \mathbf{T}^{N} \\
& R_{r(t+1)}^{N}=R_{r t}^{N}+\sum_{i \in \mathbf{I}_{r}^{+, N}} \rho_{i r}^{S T N} B_{i\left(t-\tau_{i}^{N}\right)}^{N}+\sum_{i \in \mathbf{I}_{r}^{-, N}} \rho_{i r}^{S T N} B_{i t}^{N}+\xi_{r t}^{N} \leq \psi_{r(t+1)}^{N} \\
& \beta_{i r}^{m, N} X_{i t}^{N} \leq B_{i t}^{N} \leq \beta_{i r}^{M, N} X_{i t}^{N} \quad \forall i \in \mathbf{I}^{N}, r \in \mathbf{R}_{i}^{U, N}, t \in \mathbf{T}^{N} \quad \forall r \in \mathbf{R}^{M, S T N}, t \in \mathbf{T}^{N} \backslash\left\{\eta^{N}\right\}
\end{aligned}
$$

\subsubsection{Resource-Task Network}

We use the RTN-based model first introduced by Pantelides_(Pantelides, 1994); we refer to it as model PRTN. It consists of only two equations: excess resource balance, (13), which merges (10) and (11) into a single expression, and processing unit utilization, equation (12), which is still valid for model PRTN.

$$
\begin{aligned}
& R_{r(t+1)}^{N}=R_{r t}^{N}+\sum_{i \in \mathbf{I}_{r}^{N}} \sum_{t^{\prime}=0}^{\tau_{i}^{N}}\left(\chi_{i r t^{\prime}}^{R T N} X_{i\left(t-t^{\prime}\right)}^{N}+\rho_{i r t^{\prime}}^{N} B_{i\left(t-t^{\prime}\right)}^{N}\right)+\xi_{r t}^{N} \leq \psi_{r(t+1)}^{N} \\
& \forall r \in \mathbf{R}^{N}, t \in \mathbf{T}^{N} \backslash\left\{\eta^{N}\right\}
\end{aligned}
$$

where $\chi_{i r t}^{R T N}$ is the number of discrete units of resource $r \in \mathbf{R}^{N}$ required by task $i \in \mathbf{I}_{r}^{N} t$ time points after the beginning of the task.

\subsection{Resource-Constrained Project Scheduling}

The Resource-Constrained Project Scheduling Problem (RCPSP) is a classic scheduling problem in the area of project management. It consists of a set of activities with fixed duration that must be scheduled subject to precedence and resource constraints such that a project is completed in the shortest possible time. A vast literature has been dedicated to the RCPSP, studying different variants of its three major components, namely, activities, precedence relations and resources (Brucker et al., 1999; Demeulemeester and Herroelen, 2002; Hartmann and Briskorn, 2010).

The RCPSP has also attracted the attention of researchers in PSE, because of its applications in process-related projects such as plant retrofitting and new product development. In particular, several works have applied modifications of the RCPSP to the problem of scheduling testing tasks in the development of new agricultural and pharmaceutical products (Blau et al., 2004; Colvin and Maravelias, 2010; Colvin and Maravelias, 2011; Jain and Grossmann, 1999; Papageorgiou et al., 2001; Schmidt and Grossmann, 1996; Subramanian et al., 2001). A different line of work has been devoted to derive new discrete- and continuous-time formulations for the RCPSP by extending 
concepts originally developed for the process scheduling problem (Kopanos et al., 2014; Kyriakidis et al., 2012).

A generalization of the RCPSP is the so-called Multimode RCPSP (MRCPSP), in which each activity is allowed to be executed using different modes (Talbot, 1982). Each activity mode is characterized by a different duration depending on the resource utilization level. Several efforts have been dedicated to the MRCPSP both in the OR (Weglarz et al., 2011) and PSE communities (Kyriakidis et al., 2012; Zapata et al., 2008). The MRCPSP is related to the MBPSP, since units in the same stage can be viewed as different modes of the same task. However, our derivations are based on the precedence relations of the original RCPSP. Next, we define the relevant sets and parameters for the formal definition of the RCPSP.

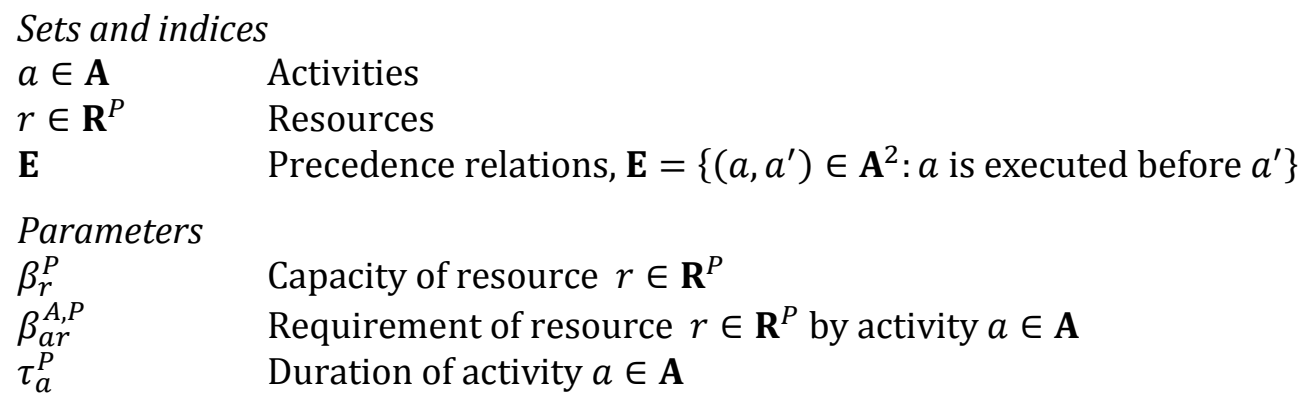

The first MIP model proposed to address the RCPSP was developed in the late 1960s (Pritsker et al., 1969). However, the limited computational capabilities at the time put a halt on the use of mathematical programming to address the RCPSP and led to the development of other exact and approximate methods (Hartmann and Briskorn, 2010). This model is now known as the aggregated discrete-time MIP formulation for the RCPSP.

Being a discrete time approach, a set of time points has to be defined. In general, the literature on RCPSP assumes that the activity durations are integer-valued so the standard step size used is one. Let $\mathbf{T}^{P}=\left\{t \in \mathbb{Z}: 0 \leq t \leq \eta^{P}\right\}$ define the set of time points, where $\eta^{P}$ is an estimate of the project duration. Two dummy activities, $a=0$ and $a=|\mathbf{A}|+1$ are added to the set $\mathbf{A}$; they represent the formal start and end of the project. In order to minimize the duration of the project, the start time of activity $|\mathbf{A}|+1$ is minimized, i.e. a makespan minimization problem is solved.

The decision to assign an activity $a$ to time period $t$ is made via a binary variable $X_{a t}^{P}, a \in \mathbf{A} \cup$ $\{0,|\mathbf{A}|+1\}, t \in \mathbf{T}^{P}$. Equations (14)-(17) define the aggregated RCPSP model. Equation (14) enforces precedence requirements by guaranteeing that the start time of a successor is greater than the finish time of a predecessor; equation (15) assigns the appropriate number of units of a resource to every activity, while satisfying resource capacity; equation (16) guarantees that every activity is executed exactly once; and equation (17) defines the objective function. This model is termed "aggregated" because the precedence constraint is only written for elements of the set $\mathbf{E}$ and the summations are made over time.

$$
\begin{aligned}
& \sum_{t \in \mathbf{T}^{P}} t X_{a^{\prime} t}^{P} \geq \sum_{t \in \mathbf{T}^{P}} t X_{a t}^{P}+\tau_{a}^{P} \quad \forall\left(a, a^{\prime}\right) \in \mathbf{E} \\
& \sum_{a \in \mathbf{A}} \sum_{t^{\prime}=t-\tau_{a}^{P}+1}^{t} \beta_{a r}^{A, P} X_{a t}^{P} \leq \beta_{r}^{P} \quad \forall t \in \mathbf{T}^{P}, r \in \mathbf{R}^{P} \\
& \sum_{t \in \mathbf{T}^{P}} X_{a t}^{P}=1 \quad \forall a \in \mathbf{A} \cup\{0,|\mathbf{A}|+1\}
\end{aligned}
$$


$\min \sum_{t \in \mathbf{T}^{P}} t X_{(|\mathbf{A}|+1) t}^{P}$

A new model was proposed in the late 1980s (Christofides et al., 1987). The only difference between the two models is in the way precedences are enforced. In this "disaggregated" model, precedence constraints are written for every pair of activities in $\mathbf{E}$ and every time point (equation (18)). This modification results in a larger formulation, but it improves its relaxation and computational performance. Equation (18) establishes that if $a, a^{\prime} \in \mathbf{A}$ are project activities such that $\left(a, a^{\prime}\right) \in \mathbf{E}$ and activity $a$ starts at time $t$ then activity $a^{\prime}$ cannot start before time $t+\tau_{a}^{P}$ and vice versa.

$\sum_{t^{\prime}=t}^{\eta^{P}} X_{a t^{\prime}}^{P}+\sum_{t^{\prime}=0}^{t+\tau_{a}^{P}-1} X_{a^{\prime} t^{\prime}}^{P} \leq 1 \quad \forall\left(a, a^{\prime}\right) \in \mathbf{E}, t \in \mathbf{T}^{P}$

The disaggregated RCPSP model is defined through equations (15)-(18).

\subsection{Solution methods for MIP models in network environments}

Models SP\&S and PRTN are relatively simple formulations. Despite their compact form, their size (i.e. number of equations and variables) is considerably larger than their continuous-time counterparts (Floudas and Lin, 2004; Mendez et al., 2006). This is the main reason why researchers in the PSE area focused on the development of continuous-time models during the 1990s and 2000s. However, as discussed in the Introduction, discrete-time models have a number of advantages. Thus, several researchers have looked into developing advanced solution methods, including methods that harness parallel computational resources (Subrahmanyam et al., 1996; Velez and Maravelias, 2013), decomposition methods (Bassett et al., 1996; Kelly and Zyngier, 2008), hybrid methods (Maravelias and Grossmann, 2004; Roe et al., 2005), reformulations (Yee and Shah, 1998), and multi-grid models (Velez and Maravelias, 2013; Velez and Maravelias, 2015).

In this paper, we are specifically interested in building upon ideas that were used by Maravelias and co-workers to develop solution methods for discrete-time models in network environments. In particular, we aim to develop reformulations (Velez and Maravelias, 2013) and algorithms for the calculation of parameters that can be used to generate tightening constraints (Velez and Maravelias, 2013; Velez et al., 2013). Interestingly, these methods were shown to be more effective when applied to discrete-time models (SP\&S and PRTN) rather than continuous-time models (Merchan and Maravelias, 2014; Merchan et al., 2013). In this section, we present an overview of the methods we will use in this paper. Full details can be found in the references provided.

\subsubsection{Reformulations}

Velez and Maravelias proposed a conceptually simple yet computationally powerful reformulation to models SP\&S and PRTN (Velez and Maravelias, 2013). They introduced an integer variable, $N_{i r}^{N}$, denoting the number of times a task $i \in \mathbf{I}^{N}$ is executed in unit $r \in \mathbf{R}_{i}^{U, N}$.

$N_{i r}^{N}=\sum_{t \in \mathbf{T}^{N}} X_{i r t}^{N} \leq\left\lfloor\frac{\eta^{N}}{\tau_{i r}^{N}}\right\rfloor \quad \forall i \in \mathbf{I}^{N}, r \in \mathbf{R}_{i}^{U, N}$

where $\tau_{i r}^{N}$ is the processing time of task $i$ in unit $r$.

Variable $N_{i r}^{N}$ characterizes any feasible schedule more effectively than a unit-disaggregated assignment variable $X_{i r t}^{N}\left(=1\right.$ if task $i$ is assigned to unit $r$ at time $t \in \mathbf{T}^{N}$ ), since multiple assignments can lead to the same number of batches processed in a unit. Therefore, it leads to a 
very effective branching. Equation (19) can be directly added to modified versions of the SP\&S and PRTN models that consider unit disaggregation. Up to four orders of magnitude reduction in CPU time was achieved. Section 5.3 discusses reformulation strategies applied to the MBPSP problem.

\subsubsection{Tightening methods}

Velez et al. proposed a family of tightening constraints for minimization problems in network environments (Velez et al., 2013). In general, the objective functions are formulated as linear combinations of the assignment variables $X_{i r t}^{N}$. When minimization problems are considered, those combinations are "pushed" to be as small as possible. The basic idea of the proposed methods was to calculate lower bounds on the production of materials based on demand information and facility characteristics. In particular, two parameters were calculated: the minimum cumulative production of tasks and materials required to satisfy customer demand.

Merchan and Maravelias extended these methods to generate tightening constraints for maximization problems in network environments (Merchan and Maravelias, 2016). In this case, the external restrictions come from initial inventory and available time. Although the maximization tries to "push" linear combinations of $X_{i r t}^{N}$ to be as large as possible, it is possible to define upper bounds on these combinations. Analogous parameters are introduced to represent the maximum cumulative production of tasks and materials attainable with the available inventory and time. A forward-propagation algorithm uses data on initial inventories for materials and processing times for tasks to calculate the corresponding parameters.

There is a fundamental difference between the approaches just described for minimization and maximization problems in network environments. While the former only considers amount-based information, the latter also uses time-based data. This distinction determines which methods can be extended to sequential environments. In the MBPSP we consider in this paper, decisions concerning amounts (i.e. the batching problem) are taken beforehand, which means that the number of batches to be scheduled is known. Thus, only the methods developed for maximization problems in network environments based on time-based data are applicable to sequential environments. Merchan and Maravelias consider the calculation of explicit time windows for each unit and task-unit combination (Merchan and Maravelias, 2016) using the following parameters:

$\varepsilon_{i r}^{U, N} \quad$ Earliest start time of task $i \in \mathbf{I}^{N}$ in unit $r \in \mathbf{R}_{i}^{U, N}$

$\varepsilon_{r}^{N} \quad$ Earliest start time of unit $r \in \mathbf{R}^{U, N}, \varepsilon_{r}^{N}=\min _{i \in \mathbf{I}_{r}^{N}} \varepsilon_{i r}^{U, N}$

$\sigma_{i r}^{U, N} \quad$ Shortest tail of task $i \in \mathbf{I}^{N}$ in unit $r \in \mathbf{R}_{i}^{U, N}$, i.e. minimum time to the end of the horizon from the end of execution of $i$ in $r$ so that it leads to final products.

$\sigma_{r}^{N} \quad$ Shortest tail of unit $r \in \mathbf{R}^{U, N}, \sigma_{r}^{N}=\min _{i \in \mathbf{I}_{r}^{N}} \sigma_{i r}^{U, N}$

$\theta_{i r}^{U, N} \quad$ Available time window for task $i \in \mathbf{I}^{N}$ in unit $r \in \mathbf{R}_{i}^{U, N}, \theta_{i r}^{U, N}=\eta^{N}-\varepsilon_{i r}^{U, N}-\sigma_{i r}^{U, N}$

$\theta_{r}^{N} \quad$ Available time window for unit $r \in \mathbf{R}^{U, N}, \theta_{r}^{N}=\eta^{N}-\varepsilon_{r}^{N}-\sigma_{r}^{N}$

The calculation of earliest start times and shortest tails is based on processing times, as well as initial inventory and unit capacities. The proposed tightening constraints are given in Equations (20) and (21).

$$
\begin{gathered}
\sum_{t \in \mathbf{T}^{N}} \tau_{i r}^{N} X_{i r t}^{N} \leq \theta_{i r}^{U, N} \quad \forall i \in \mathbf{I}^{N}, r \in \mathbf{R}_{i}^{U, N} \\
\sum_{i \in \mathbf{I}_{r}^{N}, t \in \mathbf{T}^{N}} \tau_{i r}^{N} X_{i r t}^{N} \leq \theta_{r}^{N} \quad \forall r \in \mathbf{R}^{U, N}
\end{gathered}
$$

\subsubsection{Variable time windows}


Merchan and Maravelias also proposed tightening constraints based on variable earliest start time and shortest tail (Merchan and Maravelias, 2016). Let $E_{i r}^{N}, T_{i r}^{N}$ respectively denote the actual start time and tail of task $i \in \mathbf{I}^{N}$ in unit $r \in \mathbf{R}_{i}^{U, N}$. Equations (22) and (23) establish the relation between variables and parameters defining the time windows. Additional constraints relating these new variables are defined in order to achieve the tightening effect.

$E_{i r}^{N} \geq \varepsilon_{i r}^{U, N} \quad \forall i \in \mathbf{I}^{N}, r \in \mathbf{R}_{i}^{U, N}$

$T_{i r}^{N} \geq \sigma_{i r}^{U, N} \quad \forall i \in \mathbf{I}^{N}, r \in \mathbf{R}_{i}^{U, N}$

$\sum_{t \in \mathbf{T}^{N}} \tau_{i r}^{N} X_{i r t}^{N} \leq \eta^{N}-E_{i r}^{N}-T_{i r}^{N} \quad \forall i \in \mathbf{I}^{N}, r \in \mathbf{R}_{i}^{U, N}$

Equation (24) generalizes equation (20) to variable start time and tail. In section 5.2 we discuss how these constraints are extended to sequential environments.

\section{Discrete-Time Mathematical Programming Models}

In section 2, we introduced the major constraints a feasible schedule must satisfy, namely batch precedence, batch-stage assignment, and unit assignment. This section presents four different MIP models that enforce these constraints and the different objective functions we consider. The additional sets and parameters used across the four discrete-time models are the following:

Sets

$\mathbf{K}_{i k}^{-} \quad$ Stages in which batch $i \in \mathbf{I}$ has to be processed before stage $k \in \mathbf{K}_{i}, \mathbf{K}_{i k}^{-} \equiv$ $\left\{k^{\prime} \in \mathbf{K}_{i}: k^{\prime}<k\right\}$

$\mathbf{K}_{i k}^{+} \quad$ Stages in which batch $i \in \mathbf{I}$ has to be processed after stage $k \in \mathbf{K}_{i}, \quad \mathbf{K}_{i k}^{+} \equiv$ $\left\{k^{\prime} \in \mathbf{K}_{i}: k^{\prime}>k\right\}$

Parameters

$\delta \quad$ Step size for time discretization

$\varepsilon_{j} \quad$ Earliest start time for unit $j \in \mathbf{J}$

$\varepsilon_{i j}^{U} / \varepsilon_{i k}^{S} \quad$ Earliest start time for batch $i \in \mathbf{I}$ in unit $j \in \mathbf{J}_{i}$ /on stage $k \in \mathbf{K}_{i}$

$\lambda_{j} \quad$ Latest finish time for unit $j \in \mathbf{J}$

$\lambda_{i j}^{U} / \lambda_{i k}^{S} \quad$ Latest finish time for batch $i \in \mathbf{I}$ in unit $j \in \mathbf{J}_{i}$ /on stage $k \in \mathbf{K}_{i}$

$\mu_{i} \quad$ Release date for batch $i \in \mathbf{I}, \mu_{i} \equiv\left\lceil\bar{\mu}_{i} / \delta\right\rceil$

$\tau_{i j} \quad$ Processing time for batch $i \in \mathbf{I}$ in unit $j \in \mathbf{J}_{i} \tau_{i j} \equiv\left\lceil\bar{\tau}_{i j} / \delta\right\rceil$

$\tau^{0} \quad$ Start of the scheduling horizon, $\tau^{0} \equiv \min _{i \in \mathbf{I}} \mu_{i}$

$\tau^{F} \quad$ End of the scheduling horizon, $\tau^{F} \equiv \max _{i \in \mathbf{I}} \phi_{i}$

$\phi_{i} \quad$ Due date for batch $i \in \mathbf{I}, \phi_{i} \equiv\left\lfloor\bar{\phi}_{i} / \delta\right\rfloor$

\subsection{Modeling of time}

We use index $t \in \mathbf{T}$ to denote time points. Set $\mathbf{T}$ is defined as the set of time points between the minimum release date and the maximum due date as expressed by equation (25). This horizon is divided into periods of equal length $\delta$, such that time $\bar{t}$ in the grid is given by $\bar{t}=t \delta$. We also use the elements of set $\mathbf{T} \backslash\{0\}$ to denote time periods, with period $t$ running between time points $t-1$ and $t$. Figure 2 shows the time grid we use. 
$\mathbf{T}=\left\{t \in \mathbb{Z}_{+}: \tau^{0} \leq t \leq \tau^{F}\right\}$

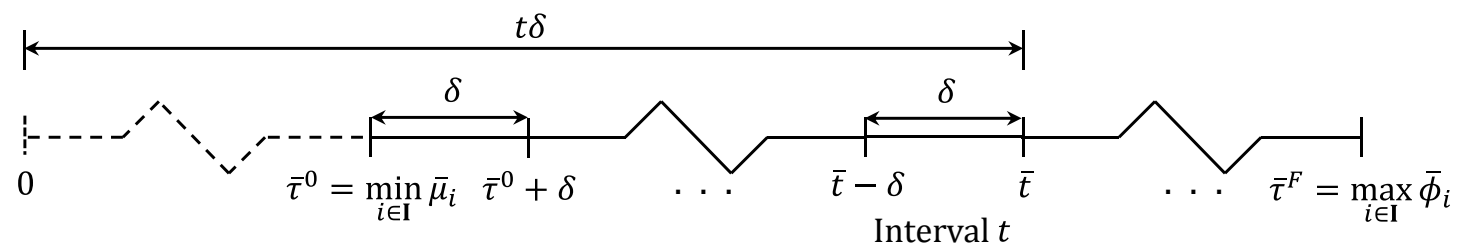

Figure 2. Global uniform grid used for discrete-time representation.

Since every batch has its own time window, which has to be implicitly enforced, we need to take into consideration the earliest start and latest finish time of a batch in specific units/stages. First, we start by defining the earliest start, $\varepsilon_{i k}^{S}$, and latest finish, $\lambda_{i k}^{S}$, times of batch $i \in \mathbf{I}$ on every stage $k \in \mathbf{K}_{i}$. The earliest batch $i$ can start on its first stage $\kappa_{i}^{F S}$ is given by its release date, $\varepsilon_{i \kappa_{i}^{F S}}^{S}=\mu_{i}$, while the latest batch $i$ can finish on its last stage $\kappa_{i}^{L S}$ is given by its due date, $\lambda_{i \kappa_{i}^{L S}}^{S}=\phi_{i}$. Equations (26) and (27) provide the definitions for all stages on which batch $i$ is processed.

$\varepsilon_{i k}^{S}=\mu_{i}+\sum_{k^{\prime} \in \mathbf{K}_{i k}^{-}} \min _{j^{\prime} \in \mathbf{J}_{i k^{\prime}}} \tau_{i j^{\prime}} \quad \forall i \in \mathbf{I}, k \in \mathbf{K}_{i}$

$\lambda_{i k}^{S}=\phi_{i}-\sum_{k^{\prime} \in \mathbf{K}_{i k}^{+}} \min _{j^{\prime} \in \mathbf{J}_{i k^{\prime}}} \tau_{i j^{\prime}} \quad \forall i \in \mathbf{I}, k \in \mathbf{K}_{i}$

We note that the earliest (latest) batch $i \in \mathbf{I}$ can start (finish) on a given unit $j \in \mathbf{J}_{i}$ only depends on the stage in which unit $j$ belongs. Equation (28) provides the definition of $\varepsilon_{i j}^{U}$, the earliest start time for batch $i$ in unit $j$, and equation (29) introduces $\lambda_{i j}^{U}$, the latest finish time for batch $i$ in unit $j$.

$\varepsilon_{i j}^{U}=\varepsilon_{i k}^{S} \quad \forall i \in \mathbf{I}, k \in \mathbf{K}_{i}, j \in \mathbf{J}_{i k}$

$\lambda_{i j}^{U}=\lambda_{i k}^{S} \quad \forall i \in \mathbf{I}, k \in \mathbf{K}_{i}, j \in \mathbf{J}_{i k}$

The concept of time window can be extended to units. Equations (30)-(31) define the earliest start time, $\varepsilon_{j}$, and latest finish time, $\lambda_{j}$ for unit $j \in \mathbf{J}$.

$\varepsilon_{j}=\min _{i \in \mathbf{I}_{j}} \varepsilon_{i j}^{U} \quad \forall j \in \mathbf{J}$

$\lambda_{j}=\max _{i \in \mathbf{I}_{j}} \lambda_{i j}^{U} \quad \forall j \in \mathbf{J}$

We can now formally define the set of feasible time points at which batch $i \in \mathbf{I}$ is allowed to start on unit $j \in \mathbf{J}_{i}$, denoted by $\mathbf{T}_{i j}$.

$\mathbf{T}_{i j}=\left\{t \in \mathbf{T}: \varepsilon_{i j}^{U} \leq t \leq \lambda_{i j}^{U}-\tau_{i j}\right\} \quad \forall i \in \mathbf{I}, j \in \mathbf{J}_{i}$

Two other sets are defined for batches and units (Castro and Grossmann, 2005). Equation (33) defines $\mathbf{I}_{j t}$, the set of batches that can start in a given unit at a specific time point, and equation (34) defines $\mathbf{J}_{i t}$, the set of units that can start processing a given batch at a specific time point.

$\mathbf{I}_{j t}=\left\{i \in \mathbf{I}_{j}: \varepsilon_{i j}^{U} \leq t \leq \lambda_{i j}^{U}-\tau_{i j}\right\} \quad \forall j \in \mathbf{J}, t \in\left[\varepsilon_{j}, \lambda_{j}\right]$

$\mathbf{J}_{i t}=\left\{j \in \mathbf{J}_{i}: \varepsilon_{i j}^{U} \leq t \leq \lambda_{i j}^{U}-\tau_{i j}\right\} \quad \forall i \in \mathbf{I}, t \in\left[\mu_{i}, \phi_{i}\right]$

\subsection{Batch-stage assignment}


All four discrete-time models we develop use a common assignment binary variable, $X_{i j t}$, which takes the value of one if batch $i \in \mathbf{I}$ starts being processed in unit $j \in \mathbf{J}_{i}$ at time point $t \in \mathbf{T}_{i j}$ and zero otherwise. Note that explicit task-unit disaggregation is considered, and that variable $X_{i j t}$ is not indexed over the set of stages, since the correspondence between units and stages is defined by $\mathbf{J}_{k}$. The four models use a common constraint to enforce the batch-stage assignment.

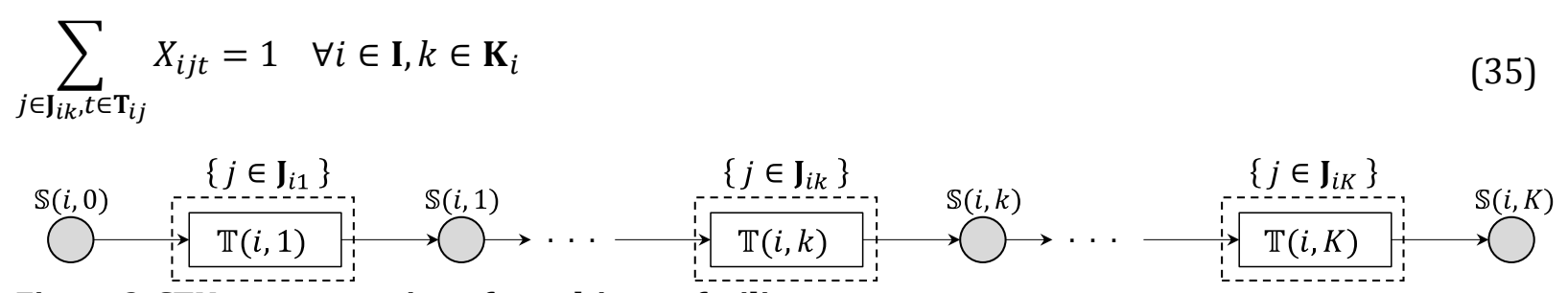

Figure 3. STN representation of a multistage facility.

Circles represent states, white boxes denote tasks, and dotted-line boxes define unit compatibility for a given task. Only a generic batch is depicted; the complete facility is represented by $I$ parallel structures. $I \equiv|\mathbf{I}|$ denotes the total number of batches and $K \equiv|\mathbf{K}|$ defines the number of stages.

\subsection{Models based on network representation}

\subsubsection{STN-based model}

As discussed in section 3.3, models based on the STN representation rely on a clique constraint for the unit assignment and a material balance for the sequencing among different units (Kondili et al., 1993; Shah et al., 1993). Although the clique constraint can readily be extended to sequential environments, the material balances cannot be expressed in terms of amounts, since they are not explicitly modeled in sequential processing. Instead, we can use the batches themselves as indicators of production of a particular batch after being processed on a given stage.

To provide an STN representation of a sequential facility, we define "task" $\mathbb{T}(i, k)$ as the processing of batch $i \in \mathbf{I}$ on any compatible unit of stage $k \in \mathbf{K}_{i}$ and state $\mathbb{S}(i, k)$ as the "material" obtained after batch $i$ has been processed on stage $k$. Figure 3 depicts the STN representation of a multistage plant. Only a general batch $i \in \mathbf{I}$ is presented in Figure 3; it is understood that the complete facility is comprised of $|\mathbf{I}|$ parallel structures such the one depicted.

The unit assignment for the STN representation of the multistage batch process is expressed through equation (36), which is a clique constraint over time and is defined on the time window of unit $j \in \mathbf{J}$.

$\sum_{i \in \mathbf{I}_{j}} \sum_{t^{\prime}=t-\tau_{i j}+1}^{t} X_{i j t^{\prime}} \leq 1 \quad \forall j \in \mathbf{J}, t \in\left[\varepsilon_{j}, \lambda_{j}\right]$

Batch precedence is enforced implicitly by requiring batch balance satisfaction at each time point. Let us define $S_{i k t}$ as a binary variable to indicate whether or not material $\mathbb{S}(i, k)$ is present during period $t \in \mathbf{T} \backslash\left\{\tau^{0}\right\}$. The batch balance expressed in equation (37) requires that a batch is present during period $t+1$ only if it was present during period $t$ or if it is produced at time point $t$. It also requires that the batch is not present during period $t+1$ if it is consumed at time point $t$. It is further assumed that material $\mathbb{S}(i, k)$ is not present at the beginning of the horizon.

$S_{i k(t+1)}=\left.S_{i k t}\right|_{t>\tau^{0}}+\sum_{j \in \mathbf{J}_{i\left(t-\tau_{i j}\right)} \cap \mathbf{J}_{k}} X_{i j\left(t-\tau_{i j}\right)}-\sum_{j \in \mathbf{J}_{i t} \cap \mathbf{J}_{k+v_{i k}}} X_{i j t} \quad \forall i \in \mathbf{I}, k \in \mathbf{K}_{i}, t \in \mathbf{T} \backslash\left\{\tau^{F}\right\}$

The STN-based model, hereinafter referred to as model M1, consists of equations (35), (36), and (37) plus the objective function. 


\subsubsection{RTN-based model}

Castro and Grossmann extended the RTN representation to sequential environments (Castro and Grossmann, 2005; Castro and Grossmann, 2006). The batch balance introduced in equation (37) remains valid to enforce batch precedence, but now a processing unit balance needs to be included to guarantee the unit assignment is satisfied. The RTN representation of a sequential process requires the definition of tasks that explicitly include the unit in which they are being executed. Let $\mathbb{T}(i, j)$ be the task defined when batch $i \in \mathbf{I}$ is processed in unit $j \in \mathbf{J}_{i}$. There are now two types of resources: states $\mathbb{S}(i, k)$ defined the same way they were introduced for the STN representation, and units $\mathbb{U}_{j}: j \in \mathbf{J}$. Figure 4 shows the RTN representation of a multistage plant. Similarly to Figure 3 , only a general batch $i \in \mathbf{I}$ is presented in Figure 4; it is understood that the complete facility is comprised of $|\mathbf{I}|$ parallel structures such the one depicted.

Unit assignment is explicitly enforced through a balance on the resource "processing units." Let $R_{j t}$ be a binary variable denoting if unit $j \in \mathbf{J}$ is available during time period $t \in \mathbf{T} \backslash\left\{\tau^{0}\right\}$. Equation (38) expresses that unit $j$ is available during period $t+1$ if it was available during period $t$ or if it is released by any task at time $t$. This balance also enforces that the unit becomes unavailable if any task starts being processed at time $t$. At the beginning of the horizon all the units are available.

$R_{j(t+1)}=\left.1\right|_{t=\tau^{0}}+\left.R_{j t}\right|_{t>\tau^{0}}+\sum_{i \in \mathbf{I}_{j\left(t-\tau_{i j}\right)}} X_{i j\left(t-\tau_{i j}\right)}-\sum_{i \in \mathbf{I}_{j t}} X_{i j t} \quad \forall j \in \mathbf{J}, t \in \mathbf{T} \backslash\left\{\tau^{F}\right\}$

The RTN-based model defined by equations (35), (37), and (38) plus the objective function is referred to as model M2 in the following.

\subsection{RCPSP inspired models}

Although a different problem when compared to the MBPSP, the RCPSP introduces an interesting way to enforce precedence constraints between a specific activity and its successors. The underlying idea is the direct calculation of times at which an activity ends and its successors start. We make the following observations to relate elements of the RCPSP and the MBPSP:

(i) There exists a unique correspondence between a project activity $a \in \mathbf{A}$ on the RCPSP and the task of processing batch $i \in \mathbf{I}$ in any compatible unit of stage $k \in \mathbf{K}_{i}$ on the MBPSP. This task is represented as $\mathbb{T}(i, k)$ like it was done for the STN representation.

(ii) The fact that a pair of activities $a$ and $a^{\prime}$ belong to the set of precedence constraints E, i.e. $\left(a, a^{\prime}\right) \in \mathbf{E}$, translates into the fact that a batch requires to be processed in consecutive stages, i.e. there is a precedence requirement for $\left(\mathbb{T}(i, k), \mathbb{T}\left(i, k+v_{i k}\right)\right)$.

(iii) Resources $r \in \mathbf{R}^{P}$ on the RCPSP correspond to processing units $j \in \mathbf{J}$ on the MBPSP. Therefore for the MBPSP the capacity of resource $r$ is $\beta_{r}^{P}=1$, and the requirement for resource $r$ by activity $a \in \mathbf{A}, \beta_{a r}^{A, P}$, is also one since task $\mathbb{T}(i, k)$ requires one processing unit.

(iv) The main difference between the RCPSP and the MBPSP is that the latter requires an additional decision for the assignment of a batch to a specific processing unit. This distinction introduces the need for appropriate unit aggregation in the constraints. Note, for instance, the difference between equations (16) and (35): an extra summation is required in (35) to account for the assignment to different units. 


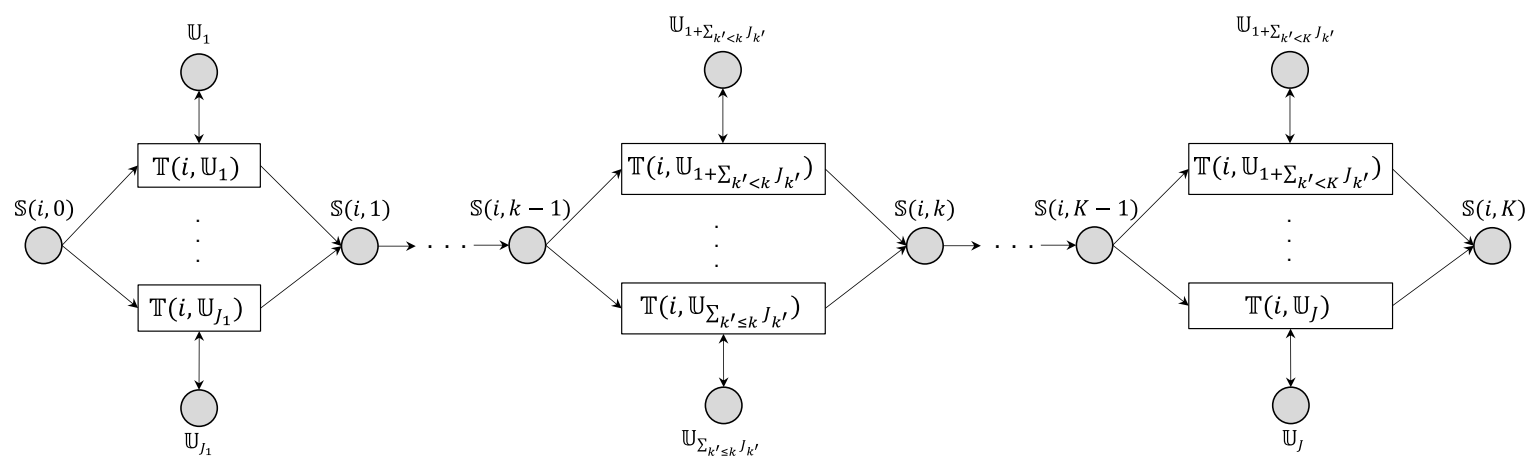

Figure 4. RTN representation of a multistage facility.

Circles represent resources (materials and units) and boxes denote tasks. Only a generic batch is depicted; the complete facility is represented by $I \equiv|\mathbf{I}|$ parallel structures; $J \equiv|\mathbf{J}|$ is the total number of units, $K \equiv|\mathbf{K}|$ is the number of stages, and $J_{k} \equiv\left|\mathbf{J}_{k}\right|$ is the total number of units on stage $k$.

Observation (iii) immediately leads to an RCPSP-based expression for the unit assignment in the MBPSP. If we substitute $\beta_{a r}^{A, P}=1$ and $\beta_{r}^{P}=1$ into equation (15), we obtain a clique over $t$, an expression completely analogous to equation (36) which enforces unit assignment for model M1. We conclude that the clique constraint in MBPSP is a simplification of the resource utilization constraint in RCPSP. Consequently, we only need to focus on developing a constraint to enforce batch precedence for the MBPSP. Observations (ii) and (iv) are the key to obtain such constraint.

\subsubsection{Aggregated formulation}

Conceptually, we want to derive a constraint which guarantees that the start time of a batch is larger than the finish time of its immediate predecessor. Equation (14) for the RCPSP can be transformed into a batch precedence constraint for the MBPSP, by taking into consideration observations (i), (ii), and (iv).

The binary variable $X_{a t}^{P}$ for activity $a \in \mathbf{A}$ in the RHS summation of equation (14) for the RCPSP corresponds to variable $X_{i j t}$ for task $\mathbb{T}(i, k)$ in the MBPSP, i.e. the processing of batch $i \in \mathbf{I}$ in any unit $j \in \mathbf{J}_{i k}$. The fact that the unit is arbitrary means that a summation over $j \in \mathbf{J}_{i k}$ must be introduced to ensure that only one $X_{i j t}$ is equal to one to calculate the finish time of $\mathbb{T}(i, k)$. Similarly, variable $X_{a^{\prime} t}^{P}$ for activity $a^{\prime} \in \mathbf{A}$ in the LHS summation of equation (14) becomes variable $X_{i j t}$ for task $\mathbb{T}\left(i, k+v_{i k}\right)$, the execution of batch $i \in \mathbf{I}$ in any unit $j \in \mathbf{J}_{i\left(k+v_{i k}\right)}$. Once again, only one $X_{i j t}$ should be nonzero when calculating the start time of $\mathbb{T}\left(i, k+v_{i k}\right)$ and thus aggregation over $j \in \mathbf{J}_{i\left(k+v_{i k}\right)}$ is required. The final form of the MBPSP batch precedence constraint is given by equation (39); it expresses that the start time of task $\mathbb{T}\left(i, k+v_{i k}\right)$ is greater than or equal to the finish time of its predecessor $\mathbb{T}(i, k)$. 


$$
\sum_{j \in \mathbf{J}_{i\left(k+v_{i k}\right)}, t \in \mathbf{T}_{i j}} t X_{i j t} \geq \sum_{j \in \mathbf{J}_{i k}, t \in \mathbf{T}_{i j}}\left(t+\tau_{i j}\right) X_{i j t} \quad \forall i \in \mathbf{I}, k \in \mathbf{K}_{i} \backslash\left\{\kappa_{i}^{L S}\right\}
$$

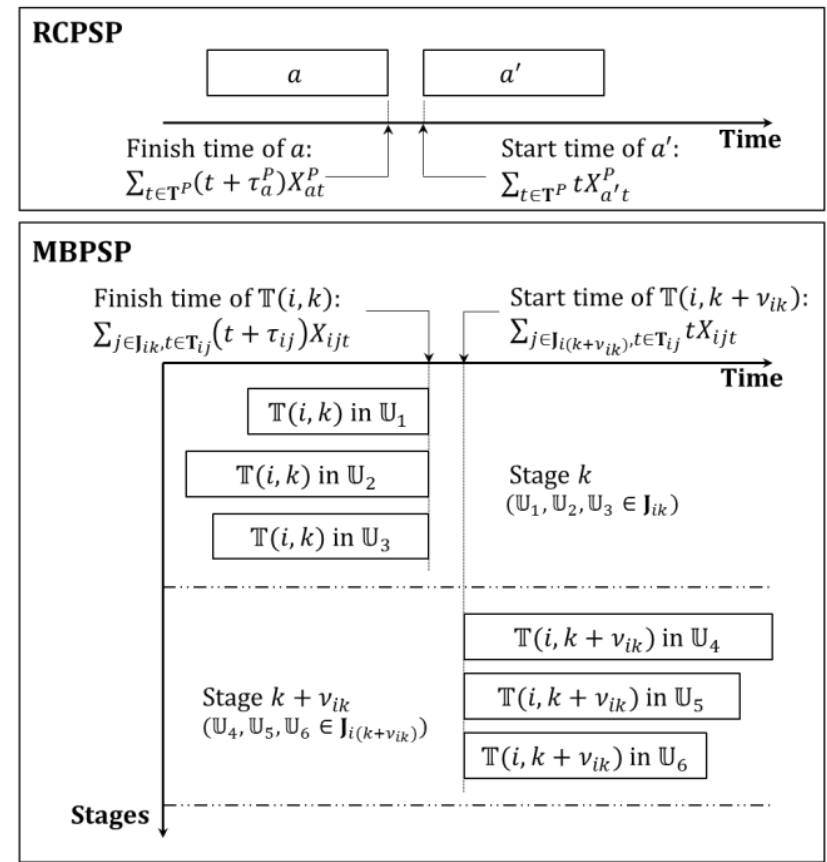

Figure 5. Time-aggregated RCPSP-based modeling of batch precedence constraints for MBPSP.

Top and bottom boxes respectively represent precedence constraints for the RCPSP and the MBPSP. Note that the finish time of task $\mathbb{T}(i, k)$ and the start time of task $\mathbb{T}(i, k)$ are independent of the processing times of batch $i$ on different units.

The aggregation over compatible units (see observation (iv)) ensures that the batch precedence relation is independent of the actual unit assignment. Figure 5 shows the correspondence between elements of the RCPSP and MBPSP that leads to equation (39). The formulation consisting of equations (35), (36), and (39) plus the objective function is henceforth referred to as model M3.

\subsubsection{Disaggregated formulation}

Our goal is to derive an expression analogous to equation (18), in order to establish a precedence between the processing of a batch in consecutive stages. We start by modifying equation (18) in order to make it extendable to the MBPSP when processing units are introduced. The second term on the LHS of equation (18) only includes decision variables for the successor activity $a^{\prime}$; however, the upper limit of this summation depends on the processing time for the predecessor activity $a$. This coupling prevents the unit aggregation required by observation (iv) above.

If $a, a^{\prime} \in \mathbf{A}$ are project activities and $\left(a, a^{\prime}\right) \in \mathbf{E}$ then equation (40) establishes that if activity $a$ finishes at time $t$ then activity $a^{\prime}$ cannot start before time $t$ and vice versa. In equation (40), each term on the LHS includes information for a single activity; therefore they are decoupled and unit aggregation is possible.

$$
\sum_{t^{\prime}=t}^{\eta^{P}} X_{a\left(t^{\prime}-\tau_{a}\right)}^{P}+\sum_{t^{\prime}=0}^{t-1} X_{a^{\prime} t^{\prime}}^{P} \leq 1 \quad \forall\left(a, a^{\prime}\right) \in \mathbf{E}, t \in \mathbf{T}^{P}
$$

Since activity a corresponds to task $\mathbb{T}(i, k)$, binary variable $X_{a\left(t^{\prime}-\tau_{a}\right)}^{P}$ in the first term of the LHS corresponds to variable $X_{i j\left(t^{\prime}-\tau_{i j}\right)}$ for the processing of batch $i \in \mathbf{I}$ in any unit $j \in \mathbf{J}_{i k}$. Aggregation 
over $j \in \mathbf{J}_{i k}$ is required to guarantee there is one nonzero assignment. Similarly, activity $a^{\prime}$ relates to task $\mathbb{T}\left(i, k+v_{i k}\right)$; therefore variable $X_{a^{\prime} t^{\prime}}^{P}$ in the second term of the LHS corresponds to variable $X_{i j t^{\prime}}$ with $j \in \mathbf{J}_{i\left(k+v_{i k}\right)}$ and aggregation over the processing units is required. Equation (41) is the precedence constraint for the disaggregated RCPSP-based model in MBPSP. It expresses that if task $\mathbb{T}(i, k)$ finishes in any unit at time $t$, then its successor $\mathbb{T}\left(i, k+v_{i k}\right)$ cannot start in any unit before $t$ and vice versa. Figure 6 presents the conceptual extension that leads to equation (41), as well as an explanation of why decoupling is necessary.

$$
\begin{aligned}
& \sum_{j \in \mathbf{J}_{i k}} \sum_{t^{\prime}=t}^{\lambda_{i j}^{U}} X_{i j\left(t^{\prime}-\tau_{i j}\right)}+\sum_{j \in \mathbf{J}_{i\left(k+v_{i k}\right)}} \sum_{t^{\prime}=\varepsilon_{i\left(k+v_{i k}\right)}^{S}}^{t-1} X_{i j t^{\prime}} \leq 1 \\
& \forall i \in \mathbf{I}, k \in \mathbf{K}_{i} \backslash\left\{\kappa_{i}^{L S}\right\}, t \in\left[\varepsilon_{i\left(k+v_{i k}\right)}^{S}+1, \lambda_{i k}^{S}\right] \cap \mathbb{Z}
\end{aligned}
$$

The complete disaggregated model consists of equations (35), (36), and (41) plus the objective function. It is hereinafter referred to as model M4.

\subsection{Objective functions}

Three different objective functions are considered, namely cost, earliness, and makespan. Cost minimization is one of the main goals in scheduling. In this section we only consider processing costs, while in Section 4.6 we also consider utility costs. In addition, other types of costs such as inventory and setup costs could be easily modeled. If $\alpha_{i j}$ is the fixed cost of processing batch $i \in \mathbf{I}$ in unit $j \in \mathbf{J}_{i}$, then the definition of the cost minimization objective is,

$\min \sum_{i \in \mathbf{I}, j \in \mathbf{J}_{i}, t \in \mathbf{T}_{i j}} \alpha_{i j} X_{i j t}$

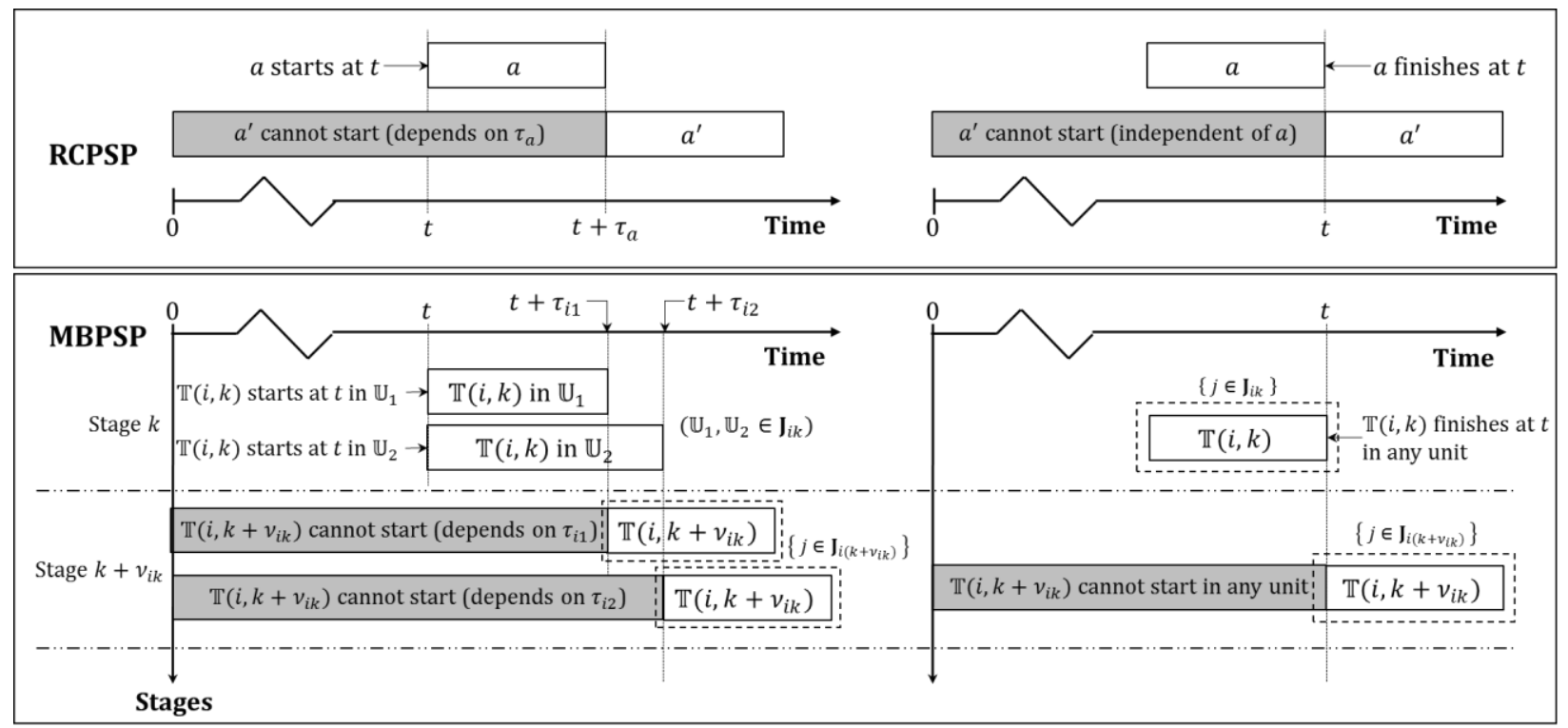

(a) No precedence decoupling

(b) Precedence decoupling

Figure 6. Time-disaggregated RCPSP-based modeling of batch precedence constraints for MBPSP

Top and bottom boxes represent precedence constraints for the RCPSP and the MBPSP, respectively. (a) Without precedence decoupling, (b) with precedence decoupling. If precedence decoupling is not used in the MBPSP (bottom left), then it is not possible to define a single window in which the successor task is not 
allowed to start. Therefore, aggregation on the units that process the predecessor task is not possible. This results in a larger and inefficient formulation for the batch precedence constraint.

Time-based objectives are used to ensure an adequate and efficient utilization of installed equipment. They also are related to customer satisfaction and inventory management. An example of the latter is earliness minimization, where the aim is to reduce storage costs. Earliness for a batch is defined as the difference between the due date and the actual finish date of that batch.

$\min \sum_{i \in \mathbf{I}}\left(\bar{\phi}_{i}-\sum_{j \in \mathbf{J}_{i \kappa_{i}^{L S}, t \in \mathbf{T}_{i j}}}\left(\delta t+\bar{\tau}_{i j}\right) X_{i j t}\right)$

The process makespan, $M S \in \mathbb{R}_{+}$, is defined as the total time it takes to complete the processing of all batches. There are several ways to define $M S$, depending on which entity of the problem the definition is based. We present three variants based on (i) batches, Equation (44); (ii) units with time disaggregation, Equation (45); and (iii) units with batch disaggregation, Equation (46).

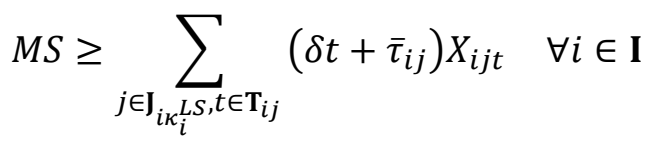

$$
\begin{aligned}
& M S \geq \sum_{i \in \mathbf{I}_{j t}}\left(\delta t+\bar{\tau}_{i j}\right) X_{i j t} \quad \forall j \in \mathbf{J}, t \in\left[\varepsilon_{j}, \lambda_{j}\right] \\
& M S \geq \sum_{t \in \mathbf{T}_{i j}}\left(\delta t+\bar{\tau}_{i j}\right) X_{i j t} \quad \forall j \in \mathbf{J}, i \in \mathbf{I}_{j}
\end{aligned}
$$

Equation (44) ensures that the makespan is larger than the finish time of every batch, while Equations (45) and (46) ensure that it is larger than the finish time of every unit.

\subsection{Limited resources}

The use of a common time-grid allows for the seamless inclusion of limited resources such as utilities and manpower. The fact that models M1-M4 employ a discrete representation of time, makes it possible to monitor variations in the utility consumption levels and model utility costs while preserving the linearity of the mathematical formulation. Sundaramoorthy and Maravelias (Sundaramoorthy and Maravelias, 2011) proposed formulations to account for utility constraints suitable for the discrete-time representation, which we incorporate into our models.

Let $r \in \mathbf{R}^{U t}$ be the set of limited utilities (e.g. cooling water, electricity, etc.) and let $\chi_{i j r}$ and $\psi_{r t}$ respectively denote the fixed requirement of utility $r$ for batch $i \in \mathbf{I}$ in unit $j \in \mathbf{J}_{i}$ and the maximum availability of utility $r$ during period $t \in \mathbf{T} \backslash\{0\}$. Furthermore, let us define decision variable $W_{r t} \in \mathbb{R}_{+}$to account for the amount of utility $r$ consumed during time period $t$. Then equation (47) provides the definition of $W_{r t}$ through a balance in terms of the total consumption of $r$ in the previous period, the consumption of $r$ by batches starting at $t$ and the release of $r$ by batches finishing at $t$.

$$
\begin{aligned}
& W_{r(t+1)}=W_{r t}+\sum_{i \in \mathbf{I}, j \in \mathbf{J}_{i t}} \chi_{i j r} X_{i j t}-\sum_{i \in \mathbf{I}, j \in \mathbf{J}_{i\left(t-\tau_{i j}\right)}} \chi_{i j r} X_{i j\left(t-\tau_{i j}\right)} \leq \psi_{r(t+1)} \\
& \forall r \in \mathbf{R}^{U t}, t \in \mathbf{T} \backslash\left\{\tau^{F}\right\}
\end{aligned}
$$

Equation (47) can be simply added to models M1-M4 whenever utilities are considered. Also, the cost minimization objective can be modified to include $\alpha_{r t}^{U t}$, the cost of utility $r$ during period $t$. 
$\min \sum_{i \in \mathbf{I}, j \in \mathbf{J}_{i}, t \in \mathbf{T}_{i j}} \alpha_{i j} X_{i j t}+\sum_{r \in \mathbf{R}^{U t}, t \in \mathbf{T}} \alpha_{r t}^{U t} W_{r t}$

Section 6 illustrates the coupling of the discrete-time models we propose with utility constraints.

\subsection{Product diversification in multistage facilities}

Hitherto we have considered that every batch processed in a sequential multistage facility defines different intermediate materials after it is processed on each stage. However, multi-stage facilities are often characterized by the transformation of a few raw materials into several different products, that is, there is a degree of diversification. For instance, a set of batches processed in a three-stage facility might share a single raw material and single intermediates after the first and second stages; only after the third stage the products are differentiated. A common example of this situation occurs when the last stage consists of packaging operations, where products are differentiated according to packaging type or size.

Traditional modeling approaches for multi-stage facilities, including the discrete-time formulations defined in section 4, treat all batches as being different from each other from the first stage of the process which might lead to unnecessarily large MIP models. The goal of this section is to show how diversification in sequential environments can be exploited to reduce formulation size.

We consider a facility where all batches share a common raw material and intermediates up to stage $|\mathbf{K}|-1$ and only the products of stage $|\mathbf{K}|$ are different. The STN representation shown in Figure 3 can be modified to account for diversification as depicted in Figure 7 . In this representation, the first $|\mathbf{K}|-1$ tasks and $|\mathbf{K}|$ states are independent of the batch and they are only indexed by $k$. Note that Figure 3 is the representation of only one generic batch; the representation of the complete facility requires $|\mathbf{I}|$ parallel structures, one for every batch. In contrast, Figure 7 represents the complete facility. When this representation change is translated into the mathematical model, a considerable reduction in number of variables and constraints can be achieved.

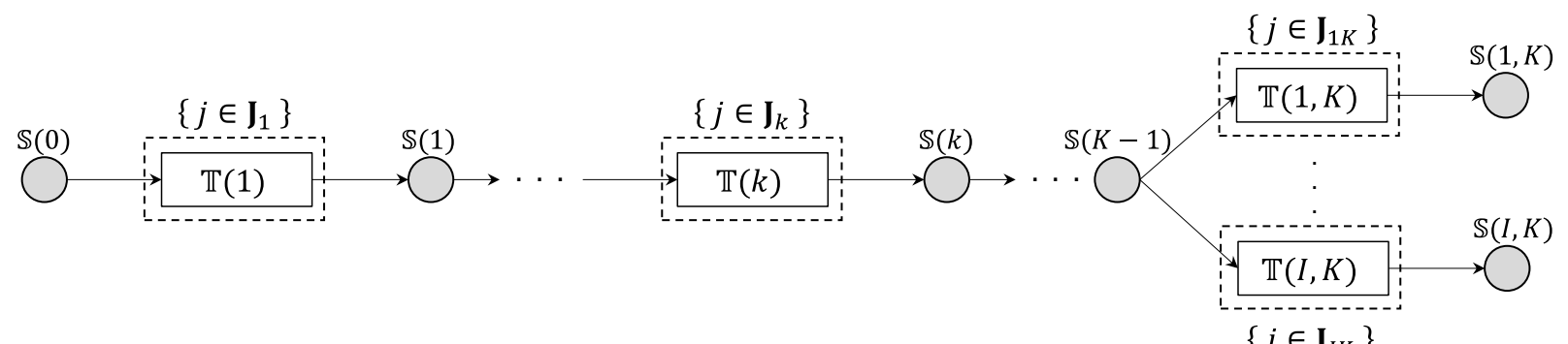

$\left\{j \in \mathbf{J}_{I K}\right\}$

Figure 7. STN representation of a multistage facility with diversification only at the last stage.

The complete facility is represented. $I \equiv|\mathbf{I}|$ denotes the total number of batches and $K \equiv|\mathbf{K}|$ defines the number of stages.

The key element to determine if a task is batch-independent is the analysis of the materials it consumes and produces. In Figure 7, we see that material $\mathbb{S}(0)$ is common to all batches, which can be formally stated as $\mathbb{S}(i, 0) \equiv \mathbb{S}(0) \forall i \in \mathbf{I}$. Similarly, $\mathbb{S}(1)$ is also common for all batches. Therefore, $\mathbb{T}(i, 1) \equiv \mathbb{T}(1) \forall i \in \mathbf{I}$. We can informally say that all tasks $\mathbb{T}(i, 1)$ collapse into a single task $\mathbb{T}(1)$.

We can generalize the concept of diversification if we consider that subgroups of tasks $\mathbb{T}(i, k)$ can be collapsed into a single task for specific subsets of batches. Let $l \in \mathbf{L}$ be an index/set to denote subgroups of batches that share common intermediates at a given stage. We can define $\mathbf{I}_{l k}$ as the $l$ th set of batches on stage $k$ for which tasks $\mathbb{T}(i, k)$ consume/produce the same set of materials. 
Informally, all the tasks $\mathbb{T}(i, k), i \in \mathbf{I}_{l k}$ collapse into a single task $\mathbb{T}(l, k)$. Unit compatibility is given by $\mathbf{J}_{l k}=\bigcap_{i \in \mathbf{I}_{l k}} \mathbf{J}_{i k}$. Figure 8 shows an example of this generalized sequential environment.

\subsubsection{Application of model SP\&S}

We now propose an STN-based model to account for diversification. Figure 8b shows that in the presence of diversification, the concepts of batch and stage are irrelevant. In fact, it is possible to reenumerate tasks/states and use model SP\&S defined for network environments.

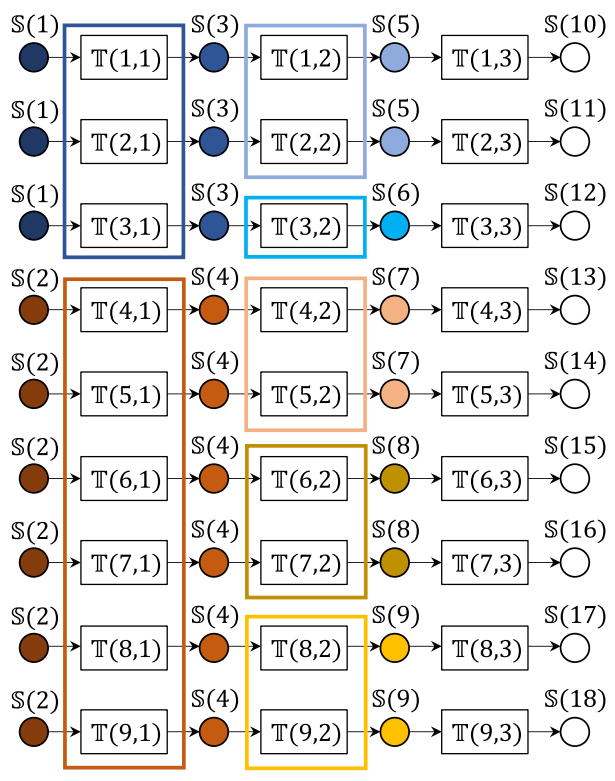

(a)

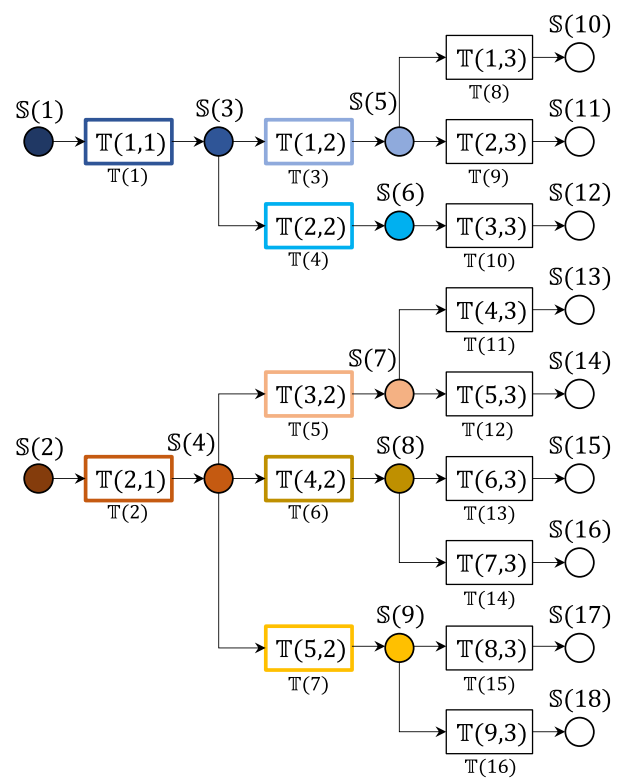

(b)

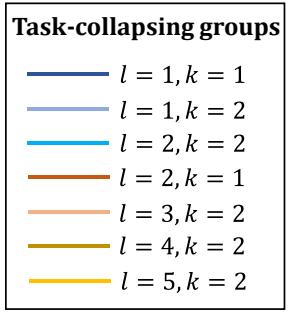

Figure 8. STN representations of a multistage facility with subgroup diversification.

Three-stage facility processing 9 batches with common intermediates. (a) Full STN representation. (b) Simplified STN representation using groups defined by diversification. In (b), the caption below every box is the re-enumeration of tasks. The color key indicates groups of tasks on (a) that collapse to a single task on (b).

After re-enumeration, task $\mathbb{T}(i)$ replaces $\mathbb{T}(l, k)$ and material $\mathbb{S}(r)$ takes the place of $\mathbb{S}(l, k)$. In this context, we use index $i \in \mathbf{I}^{N}$ to represent tasks (not the batches $i \in \mathbf{I}$, which we just dropped from the problem). We assume that inventory of raw materials $-\mathbb{S}(l, 0)$-is always available and therefore those states are not explicitly modeled. Importantly, due dates are now enforced for a set of final products, $\mathbf{R}^{M F}$, instead of the original set of orders. We denote these due dates as $\bar{\phi}_{r}^{N} \forall r \in \mathbf{R}^{M F}$.

Referring to model SP\&S we observe that (i) equation (10) can be directly used to enforce unit assignment for the MBPSP with diversification, (ii) equation (11) does not apply since amounts are not modeled in the MBPSP, and (iii) equation (12) guarantees the batch precedence requirement for the MBPSP but it must be modified to account for production/consumption of a material instead of its amount. Variable $R_{r t}^{\text {div }} \in \mathbb{Z}, r \in \mathbf{R}^{M, S T N} \backslash \mathbf{R}^{M R}, t \in \mathbf{T}^{N} \backslash\{0\}$ is nonzero if material $r$ is present during period $t$, where $\mathbf{R}^{M R}$ is the set of raw materials. The final form of the batch precedence equation is

$R_{r(t+1)}^{d i v}=R_{r t}^{d i v}+\sum_{i \in \mathbf{I}_{r}^{+, N}, j \in \mathbf{J}_{i}^{N}} X_{i j\left(t-\tau_{i j}^{N}\right)}^{N}-\sum_{i \in \mathbf{I}_{r}^{-, N}, j \in \mathbf{J}_{i}^{N}} X_{i j t}^{N} \quad \forall r \in \mathbf{R}^{M, S T N} \backslash \mathbf{R}^{M R}, t \in \mathbf{T}^{N} \backslash\left\{\eta^{N}\right\}$

The sets $\mathbf{I}_{r}^{+, N} / \mathbf{I}_{r}^{-, N}$ can readily be defined according to Figure 8 , so that task $\mathbb{T}(i) \equiv \mathbb{T}(l, k)$ consumes material $\mathbb{S}\left(r_{1}\right) \equiv \mathbb{S}(l, k-1)$ and produces $\mathbb{S}\left(r_{2}\right) \equiv \mathbb{S}(l, k)$, for values of $i, r_{1}, r_{2}$ given by the reenumeration of tasks and states. 
Note that it is not necessary to include an explicit equation to enforce the batch-stage assignment constraint of the RCPSP. In fact, when we defined the mapping between $\mathbb{T}(i)$ and $\mathbb{T}(l, k)$, the batchstage assignment is automatically satisfied. Also, the no mixing/splitting conditions are satisfied because equation (49) accounts for batches rather than amounts. No additional equations and variables are required to enforce batch integrity (Sundaramoorthy and Maravelias, 2011; Velez and Maravelias, 2013).

Equations (50) and (51) enforce due dates and guarantee that each product is delivered.

$\sum_{i \in \mathbf{I}_{r}^{+, N}, j \in J_{i}^{S T N}, t \in \mathbf{T}^{N}}\left(t+\tau_{i j}^{S T N}\right) X_{i j t}^{N} \leq \phi_{r}^{N} \quad \forall r \in \mathbf{R}^{M F}$

$R_{r \eta^{N}}^{d i v}=1 \quad \forall r \in \mathbf{R}^{M F}$

where $\eta^{N}=\max _{r \in \mathbf{R}^{M F}} \phi_{r}^{N}$ and $\phi_{r}^{N}=\left\lfloor\bar{\phi}_{r}^{N} / \delta^{N}\right\rfloor$. The three objective functions we considered can be defined through equations (52)-(54) for cost, earliness and makespan minimization respectively.

$\min \sum_{i \in \mathbf{I}^{N}, j \in \mathbf{J}_{i}^{N}, t \in \mathbf{T}} \alpha_{i j}^{N} X_{i j t}^{N}$
$\min \sum_{r \in \mathbf{R}^{M F}} \bar{\phi}_{r}^{N}-\sum_{r \in \mathbf{R}^{M F}, i \in \mathbf{I}_{r}^{+, N}, j \in \mathbf{J}_{i}^{N}, t \in \mathbf{T}^{N}}\left(\delta^{N} t+\bar{\tau}_{i j}^{N}\right) X_{i j t}^{N}$
$\min M S: M S \geq \sum_{i \in \mathbf{I}_{j}^{T N}}\left(\delta^{N} t+\bar{\tau}_{i j}^{N}\right) X_{i j t}^{N} \quad \forall j \in \mathbf{J}^{N}, t \in \mathbf{T}^{N}$

Note that in this discussion we use the notation $\mathbf{J}^{N} \equiv \mathbf{R}^{U, N}$ and $\mathbf{J}_{i}^{N} \equiv \mathbf{R}_{i}^{U, N}$ to avoid confusion between materials and units. Equation (54) uses a time-disaggregated unit-based definition for makespan, analogous to equation (45). We could also use a product-based definition for $M S$, similar to equations (44), but we omit it here for brevity. Note that the batch-disaggregated version of $M S$, equation (46), cannot be extended, since tasks are used instead of batches. The final model for MBPSP with diversification, hereinafter denoted M1div, is comprised of equations (10), (49)-(51) and the appropriate objective function (52)-(54).

\subsubsection{Multiple orders}

Model M1div relies on the fact that each product is produced and delivered once. In fact, equations (50), (51), and (53) were derived based on this assumption. However, an order might require multiple batches of the same product or there may be multiple orders of the same product. These requirements can be modeled via the introduction of pseudo-customers that are treated as additional units, so that multiple batches of the same product are delivered to different units (Sundaramoorthy and Maravelias, 2011).

Let $\mathbf{J}^{C}$ denote the set of customers and let $\bar{\phi}_{r j}^{C}$ be the due date of material $r \in \mathbf{R}^{M F}$ for customer $j \in \mathbf{J}^{C}$. It is assumed that a single customer cannot order the same product twice; if that occurs, then an additional pseudo-customer is defined. The number of customers that are required is then equal to the maximum numbers of orders for a single product. In order to model the product-customer assignment, we introduce binary variable $Z_{r j t}^{N}$ which is equal to one if material $r \in \mathbf{R}^{M F}$ is delivered to customer $j \in \mathbf{J}^{C}$ at time $t \in \mathbf{T}^{N}$. Then equations (55) and (56) replace equations (50) and (51).

$Z_{r j t}^{N}=0 \quad \forall r \in \mathbf{R}^{M F}, j \in \mathbf{J}^{C}, t>\phi_{r j}^{C}$ 
$\sum_{t \leq \phi_{r j}^{C}} Z_{r j t}^{N}=1 \quad \forall r \in \mathbf{R}^{M F}, j \in \mathbf{J}^{C}$

where $\phi_{r j}^{C}=\left\lfloor\bar{\phi}_{r j}^{C} / \delta^{N}\right\rfloor$. The material balance in equation (49) is modified to include the deliveries to customer during the scheduling horizon according to equation (57).

$$
\begin{aligned}
R_{r(t+1)}^{d i v}=R_{r t}^{d i v}+\sum_{i \in \mathbf{I}_{r}^{+, N}, j \in \mathbf{J}_{i}^{N}} X_{i j\left(t-\tau_{i j}^{N}\right)}^{N}-\sum_{i \in \mathbf{I}_{r}^{-, N}, j \in \mathbf{J}_{i}^{N}} X_{i j t}^{N}-\sum_{j \in \mathbf{J}^{C}} Z_{r j t}^{N} & \forall r \in \mathbf{R}^{M, S T N} \backslash \mathbf{R}^{M R}, t \in \mathbf{T}^{N} \backslash\left\{\eta^{N}\right\}
\end{aligned}
$$

Equations (52) and (54) are still valid for cost and makespan minimization. Nevertheless, the definition of the earliness minimization objective has to be modified, as shown in equation (58).

$\min \sum_{r \in \mathbf{R}^{M F}, j \in \mathbf{J}^{C}} \bar{\phi}_{r j}^{C}-\sum_{r \in \mathbf{R}^{M F}, j \in \mathbf{J}^{C}} \sum_{t \leq \phi_{r j}^{C}} \delta t Z_{r j t}^{N}$

The generalized model for MBPSP with diversification and multiple orders, M1divM, is defined by equations (10), (55)-(57) and the appropriate objective function from (52),(54), or (58).

\section{Solution Methods}

\section{1. $\quad$ Tightening based on fixed time windows}

In section 4.1 we discussed how the earliest start and latest finish times of a batch are used to define a set of feasible time points so that a batch is processed between its release and due dates. In this section, we extend these concepts to develop a tightening constraint for the MBPSP.

When we consider cost and earliness minimization, the time window for a unit $j \in \mathbf{J}$ is fixed and defined as the difference between its latest finish and earliest start times, i.e. $\lambda_{j}-\varepsilon_{j}$. However, when minimizing makespan, we do not know a priori whether $\lambda_{j}>M S$ or $\lambda_{j} \leq M S$. Thus we need to introduce a new parameter for the time window of a unit, regardless of the objective considered. First, let $H$ represent the end of the horizon; it can be fixed or variable as shown in equation (59).

$H= \begin{cases}\tau^{F} & \text { if end time is fixed (min Cost, Earliness) } \\ M S & \text { if end time is variable (min Makespan) }\end{cases}$

We define the shortest tail of unit $j$, denoted by $\sigma_{j}$, as the minimum elapsed time between the finishing of the last batch processed in unit $j$ and $H$; its value depends only on the processing times in stages that come after unit $j$,

$\sigma_{j}=\min _{i \in \mathbf{I}_{j}} \sum_{k \in \mathbf{K}_{i \kappa_{j}}^{+}} \min _{j^{\prime} \in \mathbf{J}_{i k}} \tau_{i j^{\prime}}$

where $\kappa_{j}$ is the stage to which unit $j$ belongs. The time window for unit $j$ can then be defined as $H-\sigma_{j}-\varepsilon_{j}$. Note that for fixed end time $\sigma_{j}=H-\lambda_{j}$, whereas for variable end time there is no general relation between $\sigma_{j}$ and $\lambda_{j}$. Equation (61) defines a tightening constraint to enforce that the actual processing time of a unit does not exceed its time window. 
$\sum_{i \in \mathbf{I}_{j}, t \in \mathbf{T}_{i j}} \tau_{i j} X_{i j t} \leq H-\sigma_{j}-\varepsilon_{j} \quad \forall j \in \mathbf{J}$

\subsection{Tightening based on variable time windows}

Tightening methods based on variable time windows were recently introduced for network environments (Merchan and Maravelias, 2016). Earliest start time and shortest tail parameters were treated as variables used to formulate constraints that are tighter than ones employing fixed counterparts. In this section, we extend those concepts to sequential environments.

\subsubsection{Start time and tail of a batch}

Let variables $E_{i k}$ and $T_{i k}$ denote the actual start time and tail, respectively, of batch $i$ on stage $k$. Then,

$$
\begin{aligned}
E_{i k} & =\sum_{j \in \mathbf{J}_{i k}, t \in \mathbf{T}_{i j}} t X_{i j t} \quad \forall i \in \mathbf{I}, k \in \mathbf{K}_{i} \\
T_{i k} & =H-\sum_{j \in \mathbf{J}_{i k}, t \in \mathbf{T}_{i j}}\left(t+\tau_{i j}\right) X_{i j t} \quad \forall i \in \mathbf{I}, k \in \mathbf{K}_{i}
\end{aligned}
$$

Lower bounds for $E_{i k}$ and $T_{i k}$ are given by $\varepsilon_{i k}^{S}$ and $\sigma_{i k}^{S}$-equations (64) and (65). Parameter $\sigma_{i k}^{S}$ is the shortest tail of batch $i$ on stage $k$, and is defined according to equation (66). Figure 9 shows a small illustration of the relation between fixed and variable start times and tails.

$E_{i k} \geq \varepsilon_{i k}^{S} \quad \forall i \in \mathbf{I}, k \in \mathbf{K}_{i}$

$T_{i k} \geq \sigma_{i k}^{S} \quad \forall i \in \mathbf{I}, k \in \mathbf{K}_{i}$

$\sigma_{i k}^{S}=\sum_{k^{\prime} \in \mathbf{K}_{i k}^{+}} \min _{j^{\prime} \in \mathbf{J}_{i k^{\prime}}} \tau_{i j^{\prime}} \quad \forall i \in \mathbf{I}, k \in \mathbf{K}_{i}$

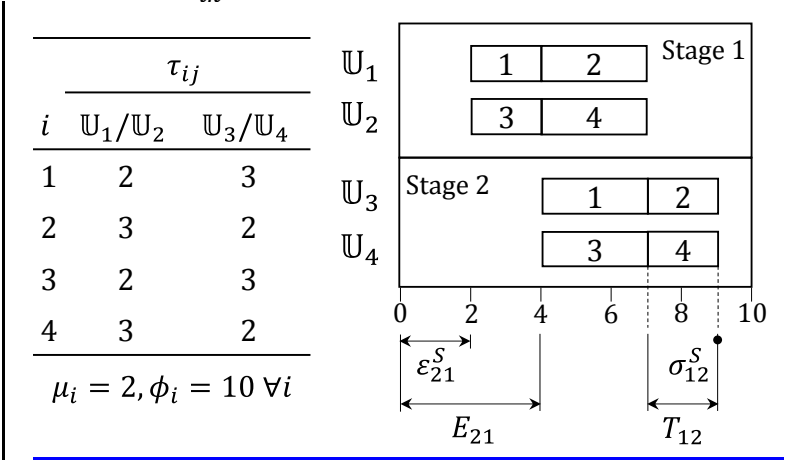

Figure 9. Start times and tails

Two-stage facility with two units per stage in which four batches are to be processed. All the batches have a common earliest start time of $\varepsilon_{i 1}^{S}=2$ for stage 1 and a shortest tail of $\sigma_{i 2}^{S}=0$ on stage 2 . The values of $E_{i k}$ and $T_{i k}$ are lower-bounded by $\varepsilon_{i k}^{S}$ and $\sigma_{i k}^{S}$ respectively, but their actual values are larger. For instance, batch 2 has an actual start time of $E_{21}=4$ on the first stage, but it could start as early as $\varepsilon_{21}^{S}=2$. Similarly, batch 1 has an actual tail of $T_{12}=2$ on the second stage but its shortest tail is $\sigma_{12}^{S}=0$.

\subsubsection{Precedence relations through start times and tails}

We can also use the variables just defined for start time and tail to explicitly enforce the sequence in which all the batches go through the stages. Equation (67) ensures that the start time of a batch on any stage exceeds the finish time of the batch on the immediately previous stage. Similarly, 
equation (68) enforces that the tail of a batch on any stage is larger than the sum of the tail and processing times of the batch on the next stages.

$$
\begin{aligned}
& E_{i\left(k+v_{i k}\right)} \geq E_{i k}+\sum_{j \in \mathbf{J}_{i k}, t \in \mathbf{T}_{i j}} \tau_{i j} X_{i j t} \quad \forall i \in \mathbf{I}, k \in \mathbf{K}_{i} \\
& T_{i k} \geq T_{i\left(k+v_{i k}\right)}+\sum_{j \in \mathbf{J}_{i\left(k+v_{i k}\right)}, t \in \mathbf{T}_{i j}} \tau_{i j} X_{i j t} \quad \forall i \in \mathbf{I}, k \in \mathbf{K}_{i}
\end{aligned}
$$

Since equations (67) and (68) relate actual time values for start and finishing of a batch in consecutive stages they can be used directly to enforce the batch precedence constraint of the MBPSP. In other words, we could write new discrete-time models by combining (i) equation (67) or equation (68)- and the definition of $E_{i k}$-or $T_{i k^{-}}$, (ii) the batch-stage assignment constraint given through equation (35), and (iii) the unit assignment constraint defined through either equation (36) or (38). In particular, equations (62) and (67) reduce to equation (39), the batch precedence constraint for model M3. In this work we study the four models described in section 4, but our formulations are flexible enough to allow the definition of up to ten different models, whose performances could be compared.

\subsubsection{Linear combinations of start times and tails}

The fact that we provide lower bounds and enforce precedence for $E_{i k}$ and $T_{i k}$ is not enough to achieve a tightening effect on the original discrete-time models. It is necessary to develop constraints that describe the dependency between start times -and between tails- of different batches. There are two cases that can be extended to MBPSP: (1) subsequent tasks, and (2) tasks that share a common unit (Merchan and Maravelias, 2016). Case (1) is already covered through equations (67) and (68), since in sequential environments the intermediate material produced by batch $i$ on stage $k$ can only be consumed by the same batch on its next stage, $k+v_{i k}$. Case (2) can directly be extended to sequential environments if we focus on the dependence of variable times for batches that share the same stage.

The method proposed by Merchan and Maravelias is based on explicitly studying all the possible sequences among tasks assigned to the same unit which is factorial in nature (Merchan and Maravelias, 2016). The result is a constraint that bounds a linear combination of these variables and can be added to the formulation. In general, units in network environments are specialized and the number of tasks assigned to a single unit remains low; thus, the exhaustive enumeration of sequences remains computationally tractable. However, in sequential environments the number of batches that can be assigned to a single stage can be significantly higher, causing a combinatorial explosion if explicit sequences were to be considered. Therefore, we adopt a different strategy to obtain the final constraints. Instead of aiming for general linear combinations for start times and tails, we focus on lower-bounding their summations as follows.

$\sum_{i \in \mathbf{I}_{k}} E_{i k} \geq \gamma_{k} \quad \forall k \in \mathbf{K}$
$\sum_{i \in \mathbf{I}_{k}} T_{i k} \geq \zeta_{k} \quad \forall k \in \mathbf{K}$

In general, lower bounds $\gamma_{k}$ and $\zeta_{k}$ are calculated by solving minimization problems, (OP1) and (OP2), subject to the original MBPSP constraints. However, in simple cases they can be estimated from simple considerations. For example, if we refer to the simple instance in Figure 9, the values of these parameters can be directed calculated as $\gamma_{1}=12(=2+4+2+4), \gamma_{2}=22(=4+7+4+$ 7), $\zeta_{1}=14(=5+2+5+2), \zeta_{2}=4(=2+0+2+0)$. 


$$
\begin{aligned}
& \gamma_{k}=\min \sum_{i \in \mathbf{I}_{k}} E_{i k} \quad \forall k \in \mathbf{K} \\
& \text { s.t. Batchstage assignment - eqn (35) } \\
& \text { Unit assignment - eqn (36) or (38) } \\
& \text { Start time definition - eqn (62) } \\
& \text { Start time bound - eqn (64) } \\
& \text { Start time sequence - eqn (67) } \\
& \zeta_{k}=\min \sum_{i \in \mathbf{I}_{k}} T_{i k} \\
& \text { s.t. Batchstage assignment - eqn (35) } \\
& \text { Unit assignment - eqn (36) or (38) } \\
& \text { Tail definition - eqn (63) } \\
& \text { Tail bound - eqn (65) } \\
& \text { Tail sequence - eqn (68) }
\end{aligned}
$$

Note that neither (OP1) nor (OP2) include batch-precedence equations (37), (39), and (41), since they are redundant with (67) or (68) as described in section 5.2.2. Moreover, computational experimentation showed that it is not necessary to solve either problem to optimality; the estimates at the root node provided very good estimates in a significantly shorter CPU time.

Equation (63), which appears as a constraint in (OP2), includes $H$ and thus depends on the objective considered. However, when solving for makespan minimization the value of $M S$ is not known, so we cannot set $H=M S$. To overcome this issue, we use a "large enough" value to estimate $M S$, and relax the due dates for all the batches, so that no extra time is unnecessarily added to the summation of tails due to the presence of early due dates. Thus, we set $H=\tau^{F}$ and $\phi_{i}=\tau^{F} \forall i \in \mathbf{I}$ when solving (OP2). Figure 10 presents a simple instance to explain why the due date relaxation is necessary.
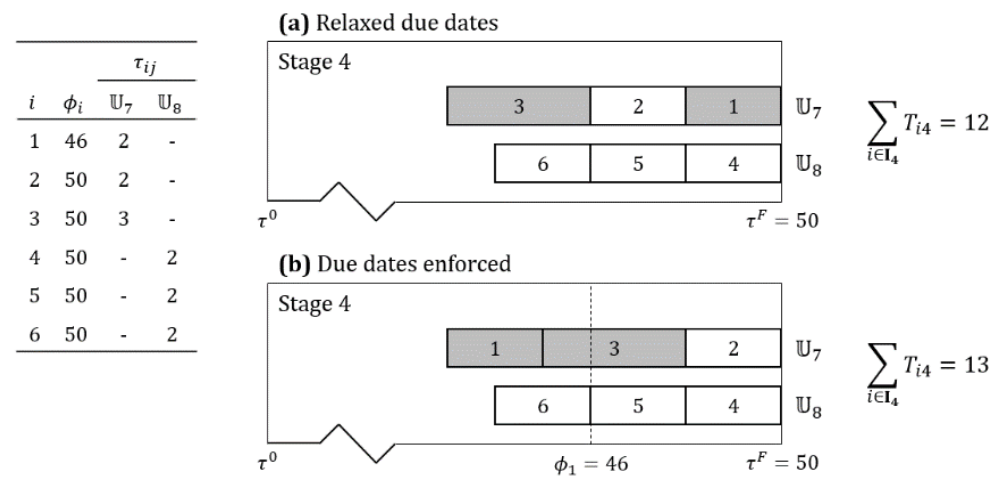

Figure 10. Due date relaxation to estimate $\zeta_{k}$.

The Gantt chart for the last stage of a four-stage facility with two units per stage is shown. Due dates, unit compatibility and processing times for six batches that need to be processed are given. (a) For relaxed due dates, batches with the smaller processing times are closer to $\tau^{F}$; therefore the summation of tails has the smallest possible value of 12 . (b) For enforced due dates, batch 1 is pushed far from $\tau^{F}$, and the summation of tails is increased to 13 . Setting $\zeta_{4}=13$ incorrectly cuts off the feasible solution in (a) for $M S \leq 46$. The correct value is $\zeta_{4}=12$.

\subsubsection{Tightening constraint}


Equation (71) is the tightening constraint that can be added to the original formulations of the four discrete-time models we proposed. It expresses that the total time batch $i \in \mathbf{I}$ spends being processed in all stages cannot exceed its available time window.

$\sum_{k \in \mathbf{K}_{i}, j \in \mathbf{J}_{i k}, t \in \mathbf{T}_{i j}} \tau_{i j} X_{i j t} \leq H-E_{i \kappa_{i}^{F S}}-T_{i \kappa_{i}^{L S}} \quad \forall i \in \mathbf{I}$

Equations (62), (63), (69)-(71) are directly added to the models to achieve the tightening effect.

\subsection{Reformulations}

As mentioned in section 3.5.1, a reformulation of the models in network environments can lead to order-of-magnitude improvements in computational efficiency. In the MBPSP, each batch $i \in \mathbf{I}$ has to be processed exactly once, regardless of the objective chosen. Thus, instead of defining the total number of batches $i \in \mathbf{I}$ assigned to a particular unit $j \in \mathbf{J}_{i}$ (which would be either zero or one), we introduce a new integer variable, $N_{j}$, denoting the total number of batches assigned to unit $j \in \mathbf{J}$.

$N_{j}=\sum_{i \in \mathbf{I}_{j}, t \in \mathbf{T}_{i j}} X_{i j t} \quad \forall j \in \mathbf{J}$

In contrast to equation (19), the summation is also performed over $i \in \mathbf{I}_{j}$, resulting in a single $j$ indexed variable. This equation can be directly added to the four discrete-time models M1-M4 presented in section 4 .

As mentioned earlier, the effectiveness of the reformulation of model SP\&S comes from the fact that branching on the new variable enables the algorithm to prune multiple assignments at once, greatly helping in terms of bound improvement. In the reformulation of models for the MBPSP, however, we note that $N_{j}$ does not exhaustively represent the solution characteristics, because different batches assigned to a unit can lead to the same value of $N_{j}$. While this might make the method less effective, the introduction of $N_{j}$ surely provides more branching options.

\subsection{Priority-based branching}

In many instances, there exists a certain stage in which the units are assigned the heaviest work load; such stage is generally known as the bottleneck stage. In these cases, the schedule of the busiest stage typically determines the schedule of the remaining stages because assignments and timing of batches in less occupied stages have more degrees of freedom compared to those the busiest stage, making it easier to adjust to the solution of the busiest stage. Exploiting this fact, we propose a method of setting priorities in the branching scheme to decision variables that are potentially more influential, namely the decision variables for the busiest stage.

In order to identify the decision variables with greater importance, we introduce variable, $G_{k}$, to quantify the busyness of a stage $k \in \mathbf{K}$. It represents the average time spent by all the units $j \in \mathbf{J}_{k}$ processing the assigned batches.

$G_{k}=\frac{1}{\left|\mathbf{J}_{k}\right|} \sum_{j \in \mathbf{J}_{k}, i \in \mathbf{I}_{j}, t \in \mathbf{T}_{i j}} \tau_{i j} X_{i j t} \quad \forall k \in \mathbf{K}$

The values of $G_{k}$ are not known a priori, because $X_{i j t}$ can only be evaluated after the problem is fully solved. However, we have found out that the LP relaxation (RMIP) of the model, which can be solved in seconds, provides a good estimate of $G_{k}$. 
We use the computed values of $G_{k}$ to identify the busiest stage, and set priorities to the decision variable of the units in that stage. Considering the reformulation from the previous section, there are two types of discrete decision variables upon which the priorities can be set: $N_{j}$ and $X_{i j t}$. We also note that the approach of setting priorities can vary. A single priority can be set to the variables related to the busiest stage, or multiple but increasing priorities can be set as the busyness of the stage increases. As a result, we study four different methods:

(i) Set priorities to $X_{i j t}$ that belongs to the stage with the highest $G_{k}$ value

(ii) Set priorities to $N_{j}$ that belongs to the stage with the highest $G_{k}$ value

(iii) Set hierarchical priorities to $X_{i j t}$ with increasing order of $G_{k}$ values

(iv) Set hierarchical priorities to $N_{j}$ with increasing order of $G_{k}$ values

\subsection{Improving the accuracy of discrete-time scheduling solutions}

One of the disadvantages of discrete-time models can be the accuracy of the solution, since all the events can only take place at the predetermined time points. To address this issue, we propose a method to improve the quality of solution obtained from discrete-time models. The method allows the refinement of solutions obtained by a discrete-time formulation using large step size $\delta$. Thus, hard instances can be solved faster with a large value of $\delta$ without any loss in accuracy. We apply this method to makespan minimization since it is the hardest objective studied in this paper.

The key idea is to solve the problem in two steps: (1) obtain assignments and sequencing through a discrete-time model, (2) refine the solution with a continuous-time model. First, the problem is solved with one of the discrete-time models presented in section 4 to obtain a solution represented in terms of $X_{i j t}$. Then, the solutions are converted into decision variables of the continuous-time model MH\&C using equations (74) through (76).

$$
\begin{aligned}
& X_{i j}^{C}=\sum_{t \in \mathbf{T}_{i j}} X_{i j t} \quad \forall i, j \in \mathbf{J}_{i} \\
& C_{i k}=\sum_{j \in \mathbf{J}_{i k}, t \in \mathbf{T}_{i j}}\left(\delta t+\bar{\tau}_{i j}\right) X_{i j t} \quad \forall i, k \in \mathbf{K}_{i} \\
& Y_{i i^{\prime} k}=1 \quad \begin{array}{l}
\forall\left(i, i^{\prime}\right) \in \mathbf{I}^{2} \quad: i<i^{\prime} \\
\forall k \in \mathbf{K}_{i} \cap \mathbf{K}_{i^{\prime}}:\left(\exists j \in \mathbf{J}_{i k} \cap \mathbf{J}_{i^{\prime} k}: X_{i j}^{C}+X_{i^{\prime} j}^{C}=2\right) \wedge\left(C_{i^{\prime} k} \geq C_{i k}+\Sigma_{j \in \mathbf{J}_{i^{\prime} k}} \tau_{i^{\prime} j} X_{i^{\prime} j}^{C}\right)
\end{array}
\end{aligned}
$$

Equation (74) calculates the continuous-time assignment variable. Equation (75) calculates completion time of a batch on a stage using aggregation of finishing times over time and units. Equation (76) ensures that the sequencing variable $Y_{i i^{\prime} k}$ is one if both $i$ and $i^{\prime}$ are assigned to the same unit and the starting time of $i^{\prime}$ exceeds the completion time of $i$.

In the next step, instead of solving the full MH\&C model, we fix the assignment and sequencing variables computed via equations (74)-(76) in equations (1) and (3)-(5), and resolve just for the timing $C_{i k}$. The algorithm in Table 1 identifies redundant constraints that can be removed from the modified MH\&C model. It calculates parameters $\omega_{i i^{\prime} j k^{\prime}}^{1} \omega_{i i^{\prime} j k^{\prime}}^{2}$, and $\omega_{i i^{\prime} j k}^{3}$ as indicators of redundant constraints: $\omega_{i i^{\prime} j k}^{1}=1$ if both batches $i$ and $i^{\prime}$ are assigned to a common unit $j \in \mathbf{J}_{i k} ; \omega_{i i^{\prime} j k}^{2}=1$ if $i$ is processed before $i^{\prime}$; and $\omega_{i i^{\prime} j k}^{3}=1$ indicates that batch $i$ is processed after $i^{\prime}$.

The modified MH\&C model, MH\&C_LP, consists of equations (6), (9) and (77)-(80).

$C_{i\left(k+v_{i k}\right)} \geq C_{i k}+\bar{\tau}_{i j} \quad \forall i \in \mathbf{I}, k \in \mathbf{K}_{i} \backslash\left\{\kappa_{i}^{L S}\right\}, j \in \mathbf{J}_{i\left(k+v_{i k}\right)}: X_{i j}^{C}=1$ 


$$
\begin{aligned}
& C_{i^{\prime} k}-\bar{\tau}_{i^{\prime} j} \geq C_{i k} \quad \forall\left(i, i^{\prime}\right) \in \mathbf{I}^{2}: i^{\prime}>i, k \in \mathbf{K}_{i} \cap \mathbf{K}_{i^{\prime}, j} \in \mathbf{J}_{i k} \cap \mathbf{J}_{i^{\prime} k^{\prime}}: \omega_{i i^{\prime} j k}^{1}=\omega_{i i^{\prime} j k}^{2}=1 \\
& C_{i k}-\bar{\tau}_{i j} \geq C_{i^{\prime} k} \quad \forall\left(i, i^{\prime}\right) \in \mathbf{I}^{2}: i^{\prime}>i, k \in \mathbf{K}_{i} \cap \mathbf{K}_{i^{\prime}, j} \in \mathbf{J}_{i k} \cap \mathbf{J}_{i^{\prime} k^{\prime}}: \omega_{i i^{\prime} j k}^{1}=\omega_{i i^{\prime} j k}^{3}=1 \\
& C_{i \kappa_{i}^{F S}} \geq \bar{\rho}_{i}+\bar{\tau}_{i j} \quad \forall i \in \mathbf{I}, j \in \mathbf{J}_{i \kappa_{i}^{F S}}: X_{i j}^{C}=1
\end{aligned}
$$

Since all the binaries are fixed, the resulting LP can be solved within seconds. As a result of this process, we obtain a schedule with a continuous timing of events and an improved objective, i.e. the refined solution. Although we note that this method does not guarantee that the optimal solution can be found, it turns out to be a powerful tool, as will be shown in section 7, in solving problems where the greatest common factor of processing time is small.

Table 1. Algorithm for elimination of redundant constraints

$$
\begin{aligned}
& \text { Set } \omega_{i i^{\prime} j k}^{1}=\omega_{i i^{\prime} j k}^{2}=\omega_{i i^{\prime} j k}^{3}=1 \quad \forall\left(i, i^{\prime}\right) \in \mathbf{I}^{2}: i^{\prime}>i, k \in \mathbf{K}_{i} \cap \mathbf{K}_{i^{\prime}, j \in \mathbf{J}_{i k} \cap \mathbf{J}_{i^{\prime} k}} \\
& \text { Loop } i \in \mathbf{I} \\
& \text { Loop } i^{\prime} \in \mathbf{I}: i^{\prime}>i \\
& \text { Loop } k \in \mathbf{K}_{i} \cap \mathbf{K}_{i^{\prime}} \\
& \text { Loop } j \in \mathbf{J}_{i k} \cap \mathbf{J}_{i^{\prime} k} \\
& \quad \text { If } X_{i j}^{C}+X_{i^{\prime} j}^{C}<2 \text { then } \omega_{i i^{\prime} j k}^{1}=0 \\
& \quad \text { If } \omega_{i i^{\prime} j k}^{1}=1 \text { and } Y_{i i^{\prime} k}=0 \text { then } \omega_{i i^{\prime} j k}^{2}=0 \\
& \text { If } \omega_{i i^{\prime} j k}^{1}=1 \text { and } Y_{i i^{\prime} k}=1 \text { then } \omega_{i i^{\prime} j k}^{3}=0
\end{aligned}
$$

End loop $(\times 4)$

\section{Illustrative examples}

\subsection{Utility constraints}

This section considers two different examples to illustrate the modeling flexibility and computational capabilities of the proposed discrete-time models. Example 1 is a modification of a small-scale literature instance (Sundaramoorthy et al., 2009). Example 2 is a medium-scale problem used to illustrate the effectiveness of our formulations as the model size increases. Complete data for these examples can be found in the Supporting Information.

We use model M4 to solve the examples in this section, but the performance of M1-M3 is similar in these instances. For a thorough comparison between different models, refer to section 7 . The instances in this section were solved using GAMS 24.4.6/CPLEX 12.6.2 on a computer with 8 GB of RAM and a dual Intel Core i7-930 processor at $2.8 \mathrm{GHz}$ running on Windows 7.

\subsubsection{Example 1}

We consider a facility with two stages and two units per stage. Twelve batches have to be processed with the objective of minimizing cost. Three different instances of this facility are considered. In the first instance, cooling water (CW) is required in both stages with a cost of $\$ 4 /($ ton $/ \mathrm{h}$ ) and a maximum availability of $7 \mathrm{ton} / \mathrm{h}$. The second instance introduces time variability on the maximum available amount of CW; initially the availability is 5 ton/h but after 20 hours it increases to 7 ton $/ \mathrm{h}$. The third instance adds periodic variability to the CW price with two levels at $\$ 5 /($ ton $/ \mathrm{h})$ and $\$ 4 /$ (ton/h) that are interchanged every 5 hours.

All three instances are modeled with 425 constraints, 37 continuous variables, and 816 binary variables. Computational results are summarized in Table 2 with the corresponding Gantt charts and utility profiles shown in Figure 11. All instances are solved relatively fast $(<45 \mathrm{sec})$ using model M4. 
We take the results of the first instance (fixed CW price and availability, Figure 11a) as reference to analyze the other two cases. When utility availability is lower during the first $20 \mathrm{~h}$ (Figure 11b), the schedule is shifted to the right so batches can be processed as late as possible, while keeping the total cost low $(<1.2 \%$ increase in cost with respect to fixed availability). When the CW price varies with time (Figure 11c), some batches are shifted back to the left so they are processed when the utility cost is low. However, the scarcity of CW at the beginning of the horizon limits the number of batches that can be shifted, and they end up being processed on periods where the price is higher. The net effect is a net cost increase ( $>7 \%$ compared to fixed cost and availability).

Table 2. Computational results for example 1, instances 1-3

\begin{tabular}{lccc}
\hline & Instance 1 & Instance 2 & Instance 3 \\
\hline CW Cost & Constant & Constant & Time-varying \\
CW Availability & Constant & Time-varying & Time-varying \\
Objective value & $1,631.00$ & $1,650.00$ & $1,747.00$ \\
MIP time (s) & $<1$ & 24.8 & 44.0 \\
\hline
\end{tabular}

\subsubsection{Example 2}

We consider a sequential facility with four stages in which 15 orders are to be processed. The first and fourth stages have three processing units each, while the second and third stages have two units. High-pressure steam (HPS) is required for stages 1 and 3; and refrigerated water (RW) is used in stages 2 and 4 . The objective is to minimize makespan. We consider two instances of this facility. The first instance assumes that there is unlimited availability for both HPS and RW. The second instance limits the amount of HPS to 10 ton/h and RW to 15 ton/h.

The unlimited availability assumption allows us to decouple the scheduling and utility assignment problems, so they can be solved in series. We use this fact to compare the performance of our models/methods with the continuous-time model MH\&C introduced in section 3.1 when solving the scheduling problem only. To solve the coupled scheduling/utility problem that results when limited resources are enforced, we used model M4 coupled with equation (61) as before. Table 4 provides model statistics and computational results for this instance. Figure 12 shows the Gantt chart and utility profiles for the optimal solution. An increase of 7 hours in makespan is necessary to accommodate the restrictions of limited HPS and RW.

Table 3 presents the results for model M4 with and without the tightening constraint based on fixed time windows, equation (61), as well as for model MH\&C. We obtained a difference in computational time of at least four orders of magnitude between models MH\&C and the tightened version of M4. Even without tightening, at least a two order-of-magnitude decrease in CPU time is observed with model M4, although it has five times more binary variables than MH\&C. 

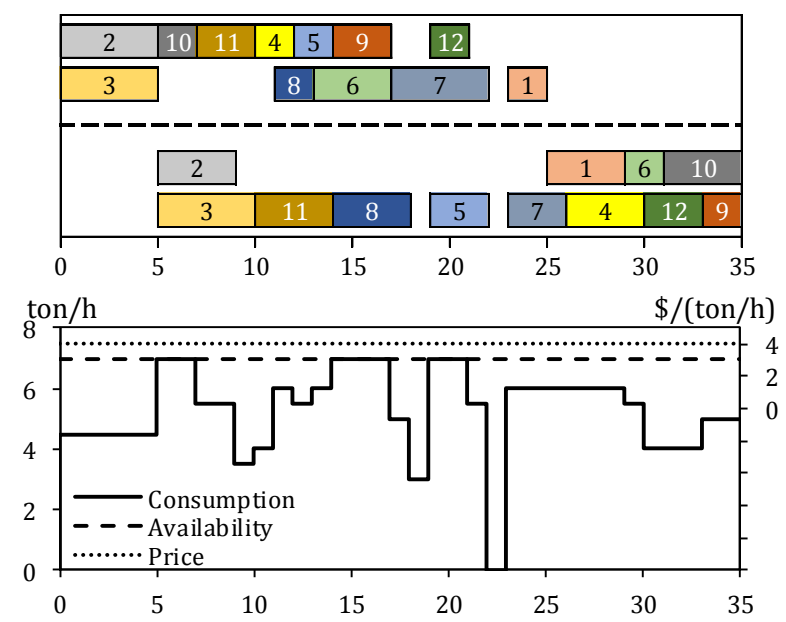

(a)
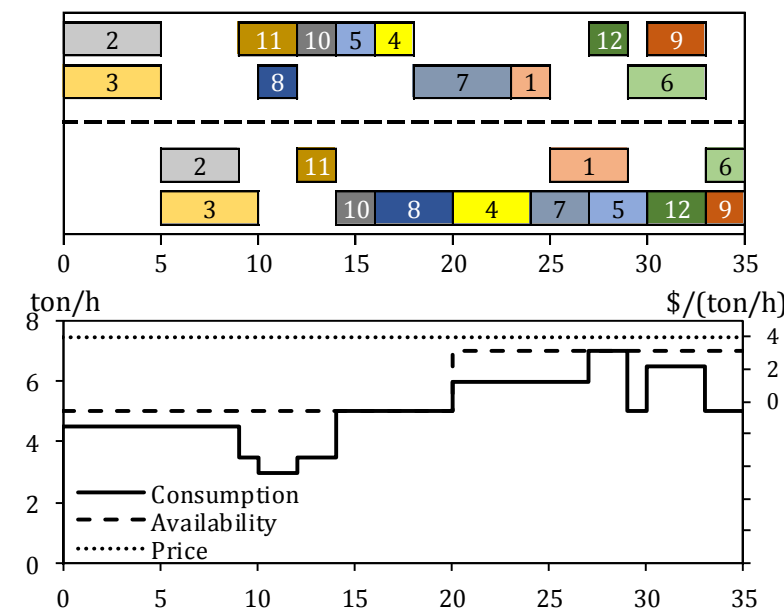

(b)
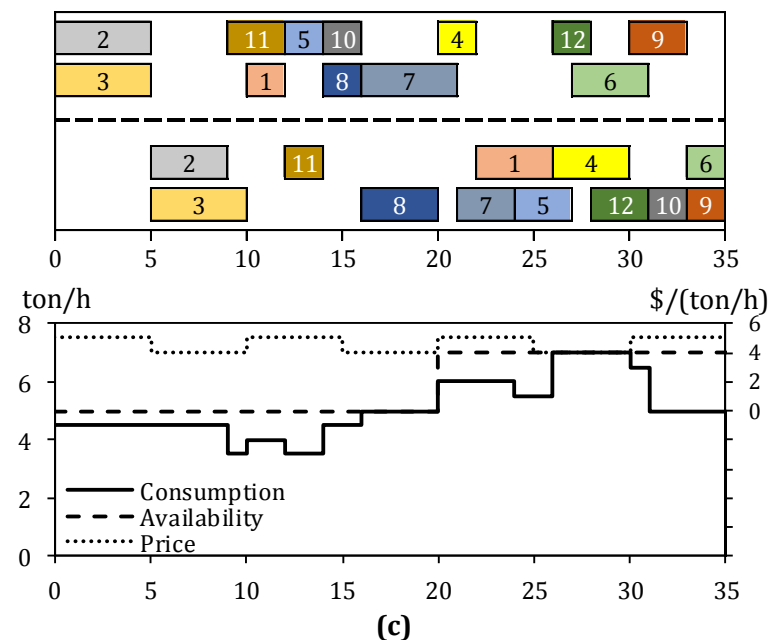

Figure 11. Gantt chart and utility profiles of optimal solutions for Example 1

(a) Constant CW cost and availability, (b) constant CW cost and time-varying availability, (c) time-varying CW cost and availability

To solve the coupled scheduling/utility problem that results when limited resources are enforced, we used model M4 coupled with equation (61) as before. Table 4 provides model statistics and computational results for this instance. Figure 12 shows the Gantt chart and utility profiles for the 
optimal solution. An increase of 7 hours in makespan is necessary to accommodate the restrictions of limited HPS and RW.

Table 3. Computational results and model statistics for example 2 with unlimited resources

\begin{tabular}{lccc}
\hline & M4 & M4+eq.(61) & MH\&C \\
\hline Constraints & 1,415 & 1,425 & 1,927 \\
Continuous variables & 1 & 1 & 56 \\
Binary variables & 2,372 & 2,372 & 480 \\
Objective value (h) & 21.00 & 21.00 & 22.00 \\
LP relaxation (h) & 2.95 & 20.00 & 13.00 \\
MIP time (s) & 134.2 & 7.0 & 10,800 \\
Nodes & 1,010 & 206 & $4,931,001$ \\
Gap (\%) & --- & --- & 12.5 \\
\hline
\end{tabular}

Table 4. Computational results and model statistics for example 2 with limited HPS and RW

\begin{tabular}{lc}
\hline & M4+eq.(61) \\
\hline Constraints & 1,589 \\
Continuous variables & 84 \\
Binary variables & 2,372 \\
Objective value (h) & 28.00 \\
LP relaxation (h) & 20.00 \\
MIP time (s) & 28.7 \\
Nodes & 1,025 \\
\hline
\end{tabular}
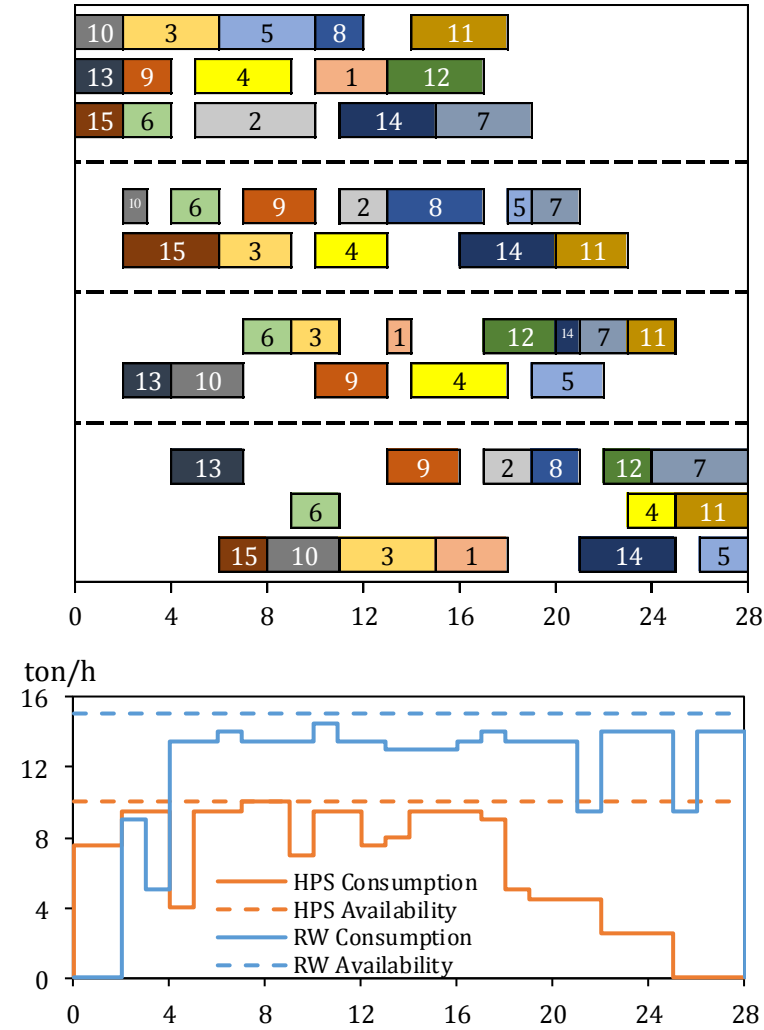

Figure 12. Gantt chart and utility profiles for Example 2 with limited resources

Fifteen batches are processed in a 4-stage facility, which uses HPS in stages 1 and 3, and RW in stages 2 and 4 . The objective is to minimize makespan with fixed utility availability. 


\subsection{Product diversification in sequential environments}

\subsubsection{Single order}

Let us consider a facility with two stages (Sundaramoorthy and Maravelias, 2011); the first stage has three units and the second has five units. Twelve products are to be processed starting from a common raw material $\mathbb{S}(0)$. The first four products have a common intermediate, $\mathbb{S}(1)$ after the first stage and use the first two units of the second stage. The next three products share $\mathbb{S}(2)$ and are compatible with the third unit of stage 2. Finally, the last five products use $\mathbb{S}(3)$ and can be processed in the last two units of the second stage. Detailed data for this facility are provided in the Supporting Information. Figure 13(a) provides a representation of this process.

We use the STN-based representation and model introduced in section 4.7 to address this problem. In this case we have three subsets of batches on stage 1: $\mathbf{I}_{11}=\{1,2,3,4\}, \mathbf{I}_{21}=\{5,6,7\}, \mathbf{I}_{31}=$ $\{8,9,10,11,12\}$. The STN representation consists of 15 tasks; the first three to transform raw material $\mathbb{S}(0)$ into common intermediates $\mathbb{S}(1)-\mathbb{S}(3)$ and the last 12 to produce individual batches from the intermediates. Figure 13(b) shows the STN representation of the process under study. Note that tasks and states have been re-enumerated, but they can be traced back to original tasks $\mathbb{T}(l, k)$ and states $\mathbb{S}(l, k)$, so the traceability of the batches is retained. For instance, $\mathbb{T}(i=3)$ corresponds to $\mathbb{T}(l=3, k=1)$ and state $\mathbb{S}(m=2)$ represents $\mathbb{S}(l=2, k=1)$.

We consider three different instances of this process, one for each objective we are studying. All three instances were solved in less than 1 CPU second. Table 5 summarizes results and model statistics.

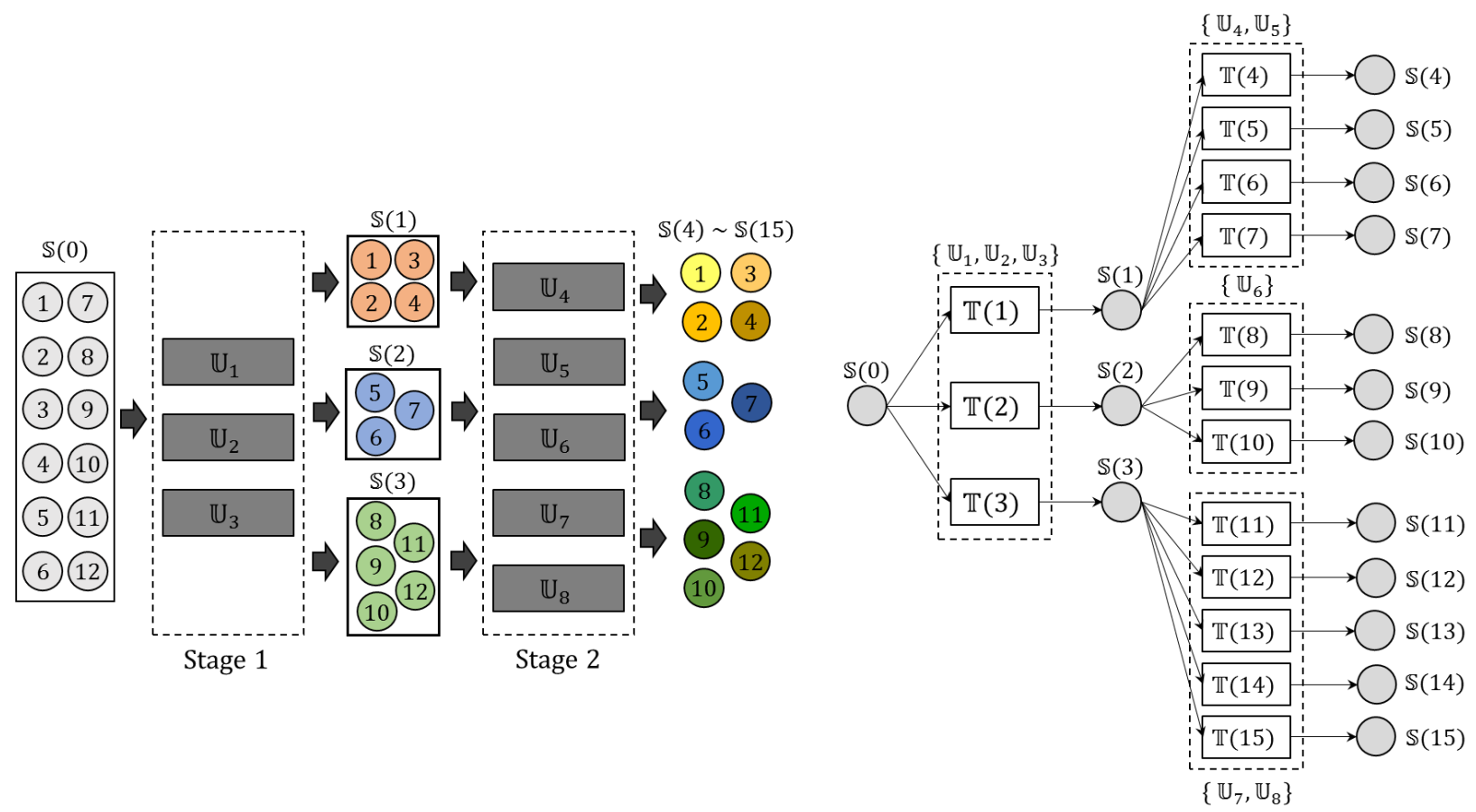

(a)

(b)

Figure 13. Sequential process with diversification.

(a) Product-stage representation of a two-stage facility where twelve products are processed. All the products share $\mathbb{S}(0)$ as raw material. Intermediates $\mathbb{S}(1), \mathbb{S}(2)$, and $\mathbb{S}(3)$ are common for three different subgroups. (b) STN representation. Stages and products are not explicitly shown. The original 12 products are represented by final products $\mathbb{S}(4)$ - $\mathbb{S}(15)$. Tasks and materials are re-enumerated. 
Table 5. Computational results and statistics for sequential process with diversification

\begin{tabular}{lccc}
\hline Model & \multicolumn{3}{c}{ M1div } \\
\hline Problem & Cost & Earliness & Makespan \\
Constraints & 741 & 741 & 753 \\
Continuous variables & 466 & 466 & 467 \\
Binary variables & 930 & 930 & 930 \\
Objective value & 955 & 0 & 14 \\
\hline
\end{tabular}

\subsubsection{Multiple orders}

We now consider the same facility depicted in Figure 13(a) when multiple orders are placed for the same product. In particular, we consider three orders of products $\mathbb{S}(4)$ - $\mathbb{S}(15)$, for a total of 36 batches. Pertinent data can be found in the Supporting Information. If we were to use a batch-based model, we would have to make three copies of the original products to consider 36 independent batches, each of them with given processing data and due dates. In contrast, using model M1divM just requires adjusting the set $\mathrm{J}^{C}$ and a single parameter $\phi_{r j}^{C}$ to reflect multiplicity of orders.

We solve the makespan minimization problem using model M1divM from section 6.2.2, as well as two more models for comparison: (1) STN-based model M1 with and without tightening, and (2) continuous-time model MH\&C. Table 6 presents the results and model statistics. It is clear that model M1divM is superior. First, there is a considerable reduction in model size; model M1divM requires $50 \%$ less constraints and $68 \%$ less binary variables (46\% less total variables) than $\mathrm{M} 1$. Second, there is an important reduction in computational time: one order of magnitude when compared with the untightened M1 and half the time after tightening, and more than three orders of magnitude when compared to MH\&C. Note that the tightened version of STN is slower than M1divM even though its relaxation is very close to the actual solution. Figure 14 presents the Gantt chart obtained for the minimum makespan problem.

Table 6. Computational results and statistics for sequential process with diversification and multiple orders

\begin{tabular}{|c|c|c|c|c|}
\hline & M1divM & & I1 & MH\&C \\
\hline Tightening & --- & No & Equation (61) & --- \\
\hline Constraints & 2,248 & 4,483 & 4,491 & 4,753 \\
\hline Continuous variables & 3,062 & 3,674 & 3,674 & 74 \\
\hline Binary variables & 1,530 & 4,731 & 4,731 & 1,008 \\
\hline Objective value (h) & 37.00 & 37.00 & 37.00 & 40.00 \\
\hline LP relaxation (h) & 6.41 & 6.41 & 36.00 & 7.00 \\
\hline MIP time (s) & 4.26 & 30.03 & 8.36 & $3,600.00^{a}$ \\
\hline Nodes & 4 & 79 & 38 & $1,112,671$ \\
\hline
\end{tabular}

a Time limit reached with optimality gap of $47.5 \%$

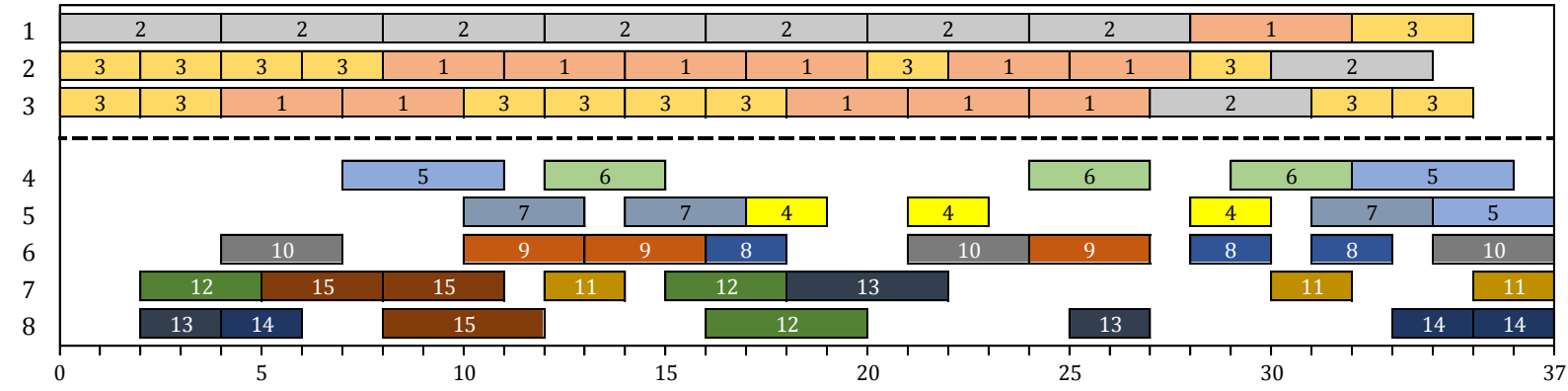

Figure 14. Gantt chart for MBPSP with makespan minimization with diversification/multiple orders. 


\section{Computational Study}

In this section, we test the performance of the four discrete-time models M1-M4 introduced in section 4, as well as the effectiveness of the solution methods proposed in section 5 using several instances of six multistage facilities. For comparison, we use model MH\&C, referred to as M5 in this section, which has proven to be one of the most effective continuous-time models for the MBPSP and has been used as a benchmark formulation in different studies (Castro and Grossmann, 2005; Castro et al., 2009; Harjunkoski and Grossmann, 2002). Next, we define the notation used in this section.

(i) Multistage facilities are identified with [P\#], where \# $\in\{1, \ldots, 6\}$. P1 is a modified version of a facility used by Harjunkoski and Grossmann (Harjunkoski and Grossmann, 2002); P2-P3 were studied by Castro and Grossmann (Castro and Grossmann, 2005); P4 appears in Liu and Karimi (Liu and Karimi, 2007); and P5-P6 are taken from Castro et al. (Castro et al., 2009). Relevant data can be found in the Supporting Information.

(ii) Objective functions are represented by [@@], where @@ $\in\{\mathrm{CT}, \mathrm{EA}, \mathrm{MS} \#\}$ for cost, earliness and makespan minimization problems, respectively. For makespan minimization we include the additional field $\# \in\{1,2,3\}$ to respectively indicate whether equation (44), (45), or (46) is used to define $M S$.

(iii) Models are denoted by $[\mathrm{M} \#]$, with $\# \in\{1, \ldots, 5\}$ as previously defined.

(iv) Inclusion of tightening constraints is identified with the pair [T\#\#], where $\# \in\{0,1\}$. The first position corresponds to the fixed-window constraint -equation (61)-, and the second position represents the variable-window constraint -equation (71)-.

(v) The use of reformulation is identified with [R\#], where \# $\in\{0,1\}$.

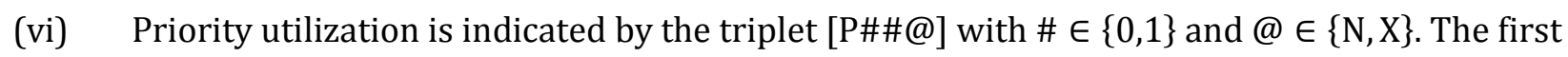
field indicates if priorities are active. The second and third field are not used if priorities are not set. The second field indicates if a branching hierarchy is used as described in section 5.4. The third field indicates if higher priorities are assigned to either the reformulation variable $N_{j}$ or the assignment variable $X_{i j t}$.

The complete notation for a single run is given by [P\#]. [@@].[M\#]. [T\#\#].[R\#].[P\#\#@]. For example, a run labeled "P3.CT.M4.T10.R1.P10N" indicates that a cost minimization (CT) problem for facility P3 is solved, using the disaggregated RCPSCP-inspired model (M4) with fixed-window tightening (T10), reformulation (R1) and higher non-hierarchical priority for the integer variable $N_{j}$ (P10N).

A total of 1,808 runs are included in this study. All the runs were solved using CPLEX 12.6.1 via GAMS 24.4 .3 on a cluster with 21 Intel Xeon (E5520) processors at $2.27 \mathrm{GHz}$ and $16 \mathrm{~GB}$ of RAM running on CentOS Linux 7. Default CPLEX options were used unless specified otherwise. A time limit of one hour was used.

We study a total of 16 formulations for each of the discrete-time models M1-M4 defined in section 4. Formulations are defined through different combinations of solution methods, i.e. different assignments of the notation fields [T\#\#].[R\#].[P\#\#@]. Table 7 shows the definition of the six basic formulations we consider. Note that priorities are only considered when the reformulation is used and that higher priorities are assigned to the reformulation variable. The cases when priorities are used without reformulation or higher priorities are assigned to assignment variables proved not to have significant impact, so they are not included in this study. The remaining ten formulations are combinations of two or three methods from F1-F5. They are denoted by [F\#.\#.\#], explicitly indicating which formulations are combined. 
Table 7. Basic formulations F0-F5

\begin{tabular}{|c|c|c|c|c|c|c|c|}
\hline \multirow{3}{*}{\begin{tabular}{l}
\multicolumn{1}{c}{ Formulation } \\
F0 \\
(Original)
\end{tabular}} & \multicolumn{2}{|c|}{ T\#\# } & R\# & \multicolumn{3}{|c|}{ P\#\#@ } & Equations \\
\hline & 0 & \multirow[t]{2}{*}{0} & \multirow[t]{2}{*}{0} & \multirow[t]{2}{*}{0} & \multirow[t]{2}{*}{--} & \multirow[t]{2}{*}{---} & M1: (35), (36), (37) \\
\hline & & & & & & & M2: (35), (37), (38) \\
\hline & & & & & & & M3: (35), (36), (39) \\
\hline & & & & & & & M4: (35), (36), (41) \\
\hline F1 & 1 & 0 & 0 & 0 & --- & --- & $\mathrm{F} 0+(61)$ \\
\hline F2 & 0 & 1 & 0 & 0 & --- & --- & F0+(61), (62), (69)-(71) \\
\hline & & & & & & & $\begin{array}{l}\text { Preprocessing: Best bound at node } 0 \text { of } \\
\text { (OP1) and (OP2) }\end{array}$ \\
\hline F3 & 0 & 0 & 1 & 0 & --- & --- & $\mathrm{F} 0+(72)$ \\
\hline $\mathrm{F} 4$ & 0 & 0 & 1 & 1 & 0 & $\mathrm{~N}$ & F0+(73)+Priority (ii) \\
\hline & & & & & & & Preprocessing: LP relaxation of F0 \\
\hline F5 & 0 & 0 & 1 & 1 & 1 & $\mathrm{~N}$ & F0+(73)+Priority (iv) \\
\hline & & & & & & & Preprocessing: LP relaxation of F0 \\
\hline
\end{tabular}

a Root-node estimate used for (OP1) and (OP2). See section 5.2.3.

\subsection{Performance analysis}

To better summarize the vast amount of results we obtained and identify trends that can be used as guidelines for our analysis, we first present performance charts for different objectives and models. A performance chart shows how efficient a formulation/model is in terms of both percentage of instances solved to optimality and relative computational time compared to the fastest formulation (Dolan and More, 2002). The abscissa corresponds to the ratio of solution times of a particular formulation/model to the fastest formulation/model. The ordinate is the fraction of instances solved to optimality within a given relative time. Figure 15 shows the best four formulations for each model-objective [M\#].[@@] combination, as well as the original formulation F0. Several trends can be observed in the subplots of Figure 15.

For earliness minimization, the original formulation F0 performs as good as the best proposed formulations/methods. This means that the original discrete-time models are good enough to minimize earliness effectively. A possible explanation for this fact lies in the relatively short CPU times required to solve this problem to optimality. Adding the constant-window tightening constraint (F1) and using reformulation (F3) appear to be the best option, although it does not outperform F0. For formulations F2 and F4, the improvement achieved with our methods is balanced with the extra time required for preprocessing. Earliness minimization proved to be the easiest objective we consider.

A very different behavior is observed for makespan minimization, which exhibits significant improvements in performance when our methods are used. An average $42 \%$ of additional instances are solved with the best formulations for each model in comparison to the original formulation. It can be observed that formulations that combine tightening constraints and reformulations appear consistently to be the best for makespan minimization. In particular, a formulation that combines F1 (constant time window tightening) and F3 (reformulation without priorities) appears in the performance chart for every model. Makespan minimization is the hardest problem with only $78 \%$ of instances solved to optimality with the best formulations within the time limit.

Cost minimization is the intermediate objective in terms of difficulty and performance improvement when our models and methods are used. Although $100 \%$ of the instances were solved to optimality within an hour using our methods, the average times are higher than those for earliness minimization. In terms of performance, we observe that formulation F0 only solves $75 \%$ of the instances within 15 times the fastest formulation. In contrast, the best of our methods solved $100 \%$ of the instances within 3 times the fastest formulation. Although a general trend in terms of 
models is not as clear as it is for the other two objectives, it can be observed that the reformulation (F3, F4 and combinations) consistently rank among the best performers.

In summary, F0 is the best formulation for earliness minimization, regardless of the model. For makespan minimization F1.3 is the best for models M1-M2, F1.2.4 is the best for M3 and F1.4 is the best for M4. For cost minimization, F4 is the best formulation for M2-M3, F3 is the best for M1 and F1.5 is the best for M4.

EA
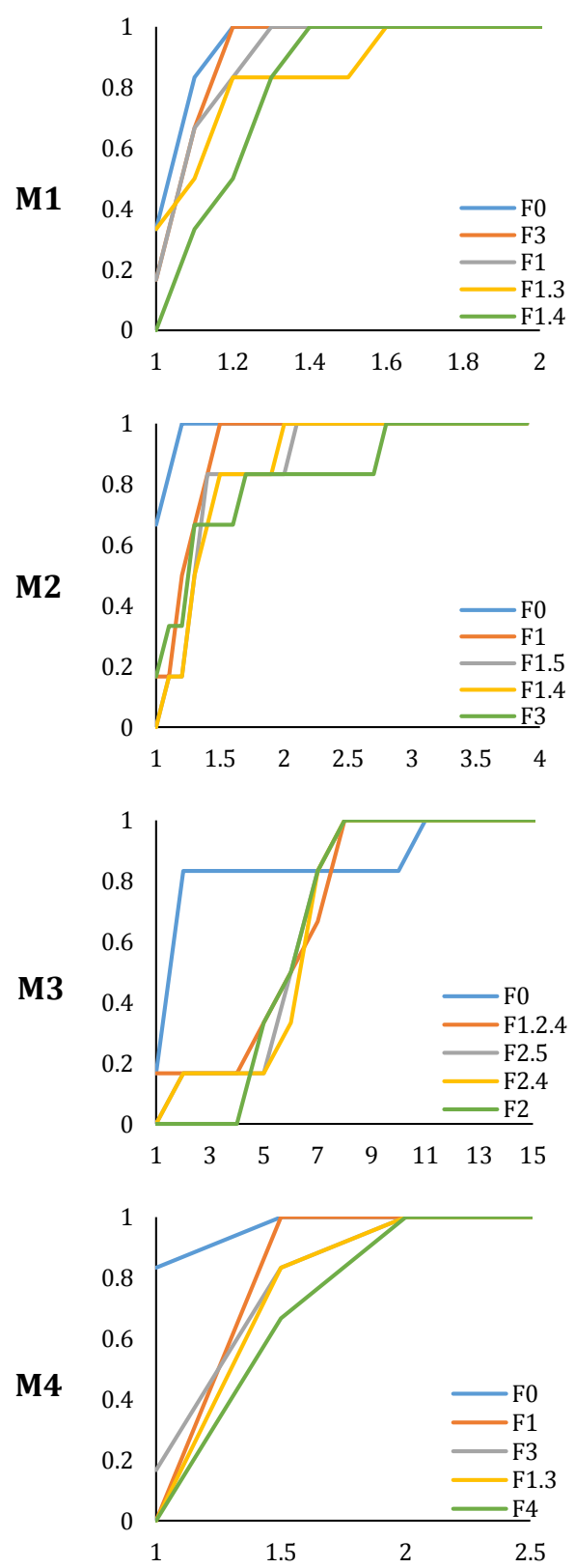

MS
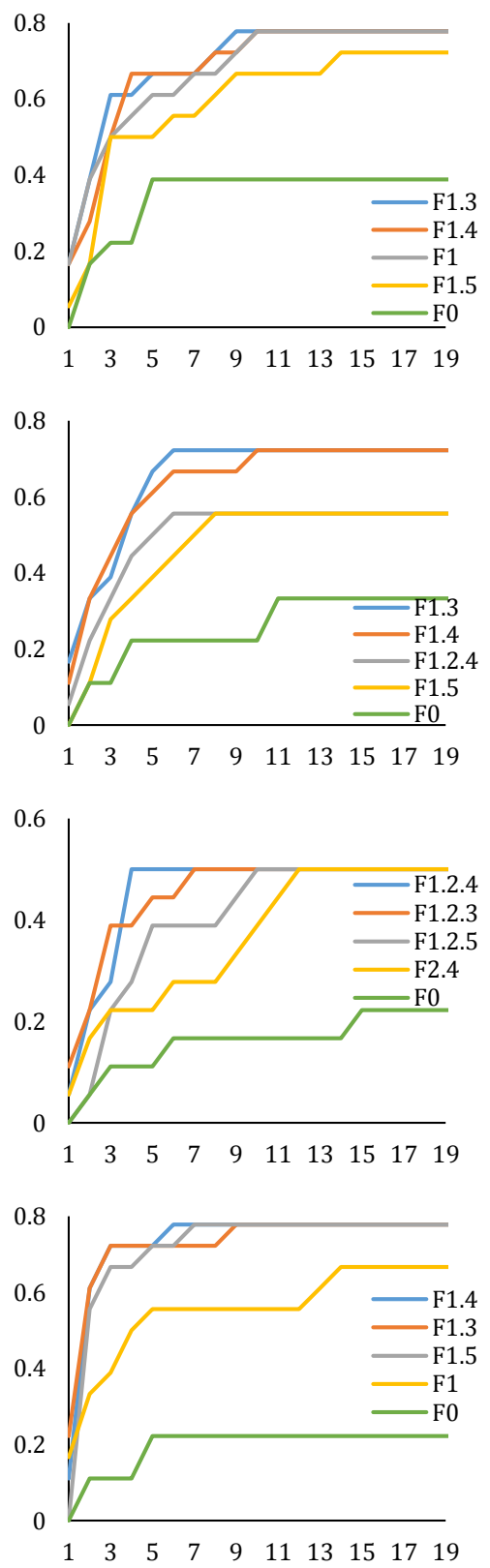

CT
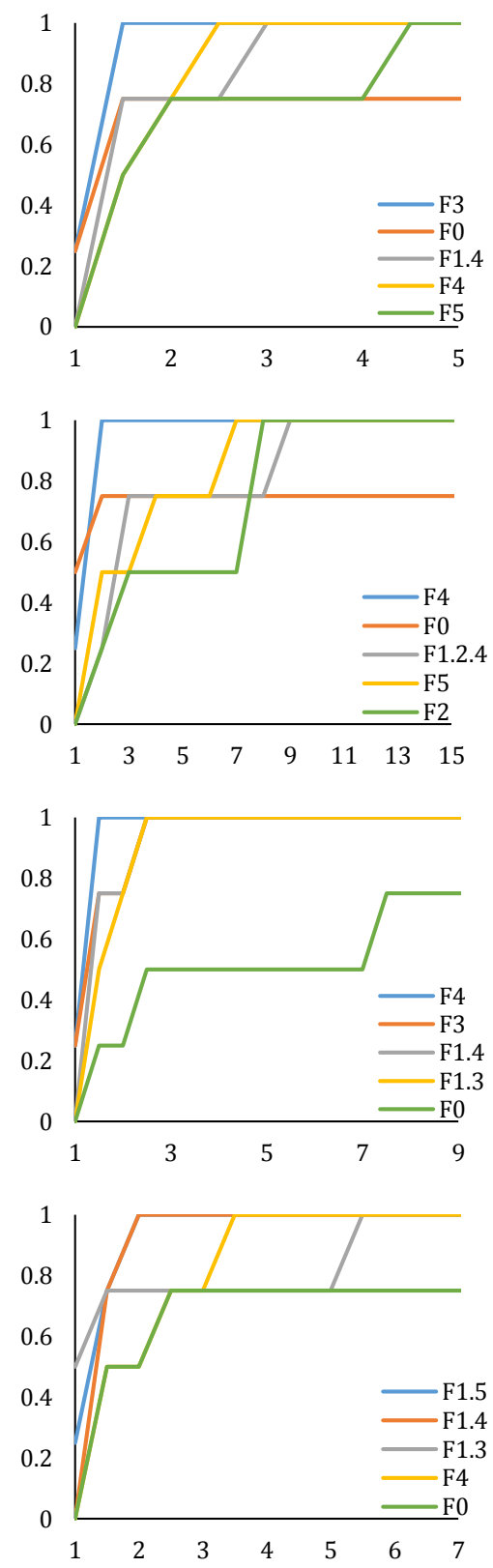

Figure 15. Performance charts of formulations for different model-objective combinations.

Combinations of $[\mathrm{M \# ]}$.[@@] are presented with \# $\in\{1, \ldots, 4\}$ and @@@ $\in\{\mathrm{CT}, \mathrm{EA}, \mathrm{MS}\}$. The legend of each subplot and the line colors indicate decreasing order of performance. The abscissa is the ratio $R=$ (CPU time)/(CPU time for fastest formulation). The ordinate is the fraction of instances solved to optimality 
in less than $R$ times the fastest instance. The legend of each subplot is listed in decreasing order of performance.

We now use performance charts to compare among different discrete-time models. We also include the continuous-time model M5 in order to show the computational capabilities of formulations currently available in the PSE literature. Figure 16 presents performance profiles for each objective considered, when the best formulation for each model is used. From Figure 16 we observe that the best model for both earliness and makespan minimization is the STN-based formulation M1. For cost minimization, however, the continuous-time formulation, model M5, produces the best results, closely followed by the aggregated RCPSP-inspired model M3. Nonetheless, we observe for the objectives where discrete-time models are superior, the performance is much better. The percentage of additional instances solved to optimality with the discrete-time models is significant: $67 \%$ for earliness and 33\% for makespan. Even if our solution methods are applied to model M5 for earliness and makespan minimization, its performance does not improve (results not shown).

EA

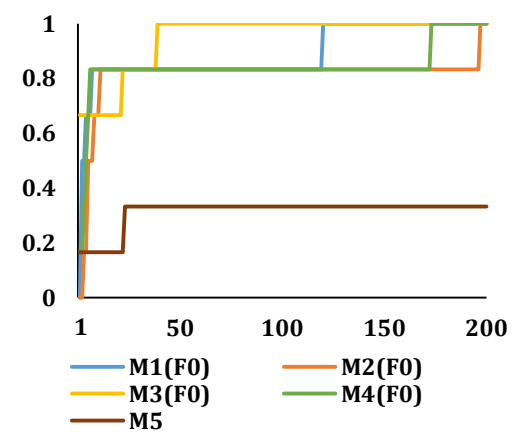

MS

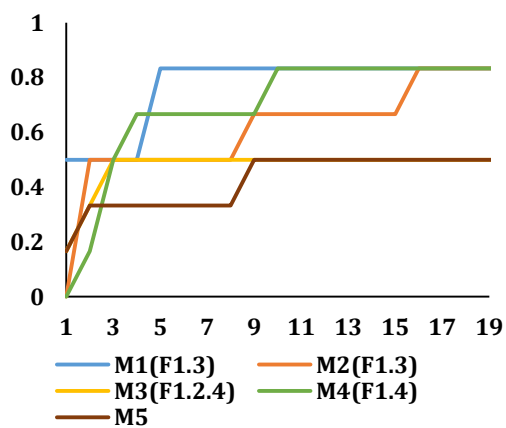

CT

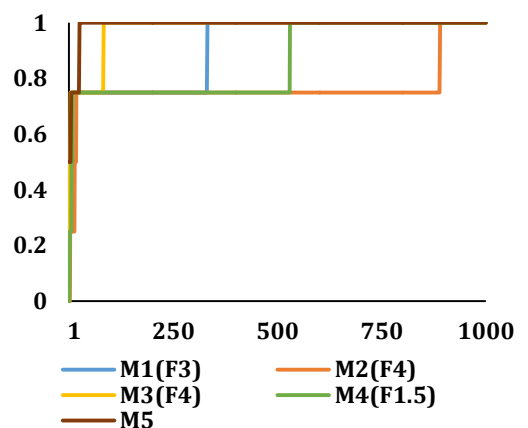

Figure 16. Performance charts of models for different objectives

Performance curves of models M1-M5 for each objective (EA: earliness, MS: makespan, CT: cost). Models M1M4 use the best formulation for a particular objective. The abscissa is the ratio $R=(C P U$ time)/ (CPU time for fastest model). The ordinate is the fraction of instances solved to optimality in less than $R$ times the fastest instance.

\subsection{Makespan minimization in hard instances}

Makespan minimization is by far the hardest objective. Therefore, additional solution techniques can be developed in order to reduce the computational burden. The next subsections provide different strategies that can be used to solve makespan minimization problems.

\subsubsection{Variable time window}

Based on the performance analysis of the previous section, formulation F2 based on variable time windows does not seem to be competitive when compared against formulations F1 (constant time window) or F3 (reformulation). However, this is the result of averaging over both small- and largescale instances. The preprocessing time required for F2 is comparable to solution times of small instances but it is much shorter than solution times of larger problems. We now focus on facilities P2, P3, P4 and P6 which are the largest instances presented in their respective references (Castro and Grossmann, 2005; Castro et al., 2009; Liu and Karimi, 2007) to show that significant improvements in computational time and optimality gap are achieved by using F2.

A total of 48 instances including these facilities are solved using different combinations of the four discrete-time models and three definitions of the makespan minimization objective. In order to perform the analysis, instances were classified in five different categories: (i) optimal solution found by both F0 and F2, (ii) optimal solution found only by F2, (iii) suboptimal solution found by 
both F0 and F2, (iv) suboptimal solution found only by F2, and (v) no solution returned for F0 nor F2. Figure 17 summarizes the results for these five categories. Clearly, formulation F2 significantly outperforms F0. When instances are solved to optimality by both formulations, F2 employs less than a third of the time F0 uses. $43.7 \%$ of the instances are solved to optimality with F2 compared to only $16.7 \%$ with $\mathrm{F} 0$. When both formulations reach the time limit without proving optimality, the gap is reduced by a third with F2. Finally, $25 \%$ of the instances did not return a feasible solution with F0, compared to only $6.7 \%$ that could not find a solution with F2. For further illustration, Table 8 presents results for four particular instances, one for each facility considered. Note that F2 is not only substantially faster but also yields, in general, solutions of much higher quality.

\begin{tabular}{|c|c|c|c|}
\hline $1,029.07$ & $30.7 \%$ & $28.3 \%$ & \\
\hline \multicolumn{4}{|l|}{ F2 } \\
\hline 324.08 & $1,346.16$ & $8.9 \%$ & $10.8 \%$ \\
\hline
\end{tabular}

Figure 17. Aggregated computational results for $\mathrm{FO}$ and $\mathrm{F} 2$ for large instances

Computational results of 48 instances solved with F0 and F2. The numbers in the box represent the average CPU time to solve to optimality, and percentiles represent the average gap.

Table 8. Computational results for one instance of each large-scale facility

\begin{tabular}{c|ccc|ccccc}
\hline \multirow{2}{*}{ Instance } & \multicolumn{3}{|c|}{ F0 } & \multicolumn{5}{c}{ F2 } \\
\cline { 2 - 8 } & CPU time (s) & Objective & Gap (\%) & Preproc. (s) & MIP time (s) & CPU time (s) & Objective & Gap (\%) \\
\hline P2.MS1.M2 & $3,600.1$ & 358 & 99.0 & 90.3 & $3,600.1$ & $3,690.4$ & 235 & 7.25 \\
P3.MS2.M1 & 3600.1 & 262 & 95.4 & 17.3 & 262.6 & 279.9 & 252 & --- \\
P4.MS3.M3 & $3,600.1$ & 162 & 14.2 & 8.5 & 555.7 & 564.2 & 162 & --- \\
P6.MS3.M4 & $3,600.0$ & NA $^{\text {a }}$ & NA $^{\text {a }}$ & 45.4 & $3,600.3$ & $3,645.7$ & 40 & 3.3 \\
\hline
\end{tabular}

a No feasible solution returned

\subsubsection{Solution refinement}

We now apply the method described in section 5.5 to a particular large-scale instance to test its effectiveness. We revisit facility P6, denoted P13 in the original reference (Castro et al., 2009), which is adapted from a real industrial case study. Hitherto, a value of $\delta=1$ is used to solve approximations of this instance, but if exact solutions were to be found, the step size would need to be $\delta=1 \times 10^{-3}$, given the values of processing times (complete data can be found in the Supporting Information). Such small value for the discretization parameter renders discrete-time models intractable; even with the value of $\delta=1$ the problem is hard to solve as discussed in the previous subsection.

The two-step method is applied to P6: (i) an optimal solution with $\delta=0.5$ is found, and (ii) model MH\&C_LP is used to refine the timing variables using the assignment and sequencing obtained by the discrete-time model. For the first step, we note that optimality cannot be proved within the original time limit if equations (44)-(46) are used to explicitly define the variable $M S$, even for small estimates of the makespan value. Instead, the iterative approach used by Castro and Grossmann is adapted to models M1-M4 (Castro and Grossmann, 2005). If we start with $\delta=1$, after the refinement we obtain a solution with a makespan of $33.615 \mathrm{~h}$. The total time to obtain this solution was only 97 seconds. If we use $\delta=0.5$, then we obtain a solution with $M S=30.376 h$ in 1,762 seconds. The best known solution to this instance is $M S=30.053 \mathrm{~h}$.

\section{Conclusions}

We presented an essentially new paradigm for the solution of scheduling problems in multiproduct multi-stage batch facilities. We discussed four MIP formulations employing discrete 
representation of time. We also developed solution methods and showed that when they are applied to the proposed models, lead to significant computational improvements thereby allowing us to address medium-size instances effectively. One of the two major advantages of these models is their simplicity and generality; they can be easily modified to account for different features common to chemical production scheduling, including utility constraints and product diversification. The other major advantage is their computational performance compared to existing approaches.

Two of the proposed formulations are based on the STN and RTN representations used primarily in network production environments. The other two are inspired by formulations for the RCPSP. In terms of solution strategies, we explored three different ideas. First, we formulated two types of tightening constraints based on time windows for both tasks and units: a constant-window-based constraint and an extension based on variable time windows. Second, we introduced a reformulation in terms of number of batches processed by each unit coupled with branching strategies using domain-specific knowledge. Third, we developed a solution refinement approach that yields more accurate solutions but can also be used to address hard instances.

We showed that the original discrete-time models are effective in solving the easiest of objectives discussed in this work, namely earliness minimization. However, a combination of methods is required to effectively reduce the solution times when makespan or cost minimization are considered. For makespan minimization, we found that the constant-window constraint combined with the reformulation has the best performance. For cost minimization, we observed that reformulation methods were more effective than the tightening constraints.

In terms of models, we found that all the proposed models outperform one of the best continuoustime models in the literature. In some cases, up to three order of magnitude reduction in computational time is achieved. For both earliness and makespan minimization, the best results were obtained with the STN-based model, M1. Cost minimization problems are addressed most effectively using the continuous-time model M5. However, discrete-time models M1 and M3 have comparable performances in term of number of instances solved to optimality and relative solution times.

Furthermore, we discussed how hard makespan minimization problems can be solved effectively. In particular, we showed how the variable-window formulation, F2, leads to significantly faster solution times and smaller optimality gaps.

Given the simplicity and generality of the proposed models and the effectiveness of the proposed solution methods, the present work lays the foundation for an alternative approach to multiproduct multi-stage batch facility scheduling. Future research directions include the modeling of general multi-purpose facilities, the treatment of complex storage constraints, and the simultaneous batching and scheduling in sequential production environments. 


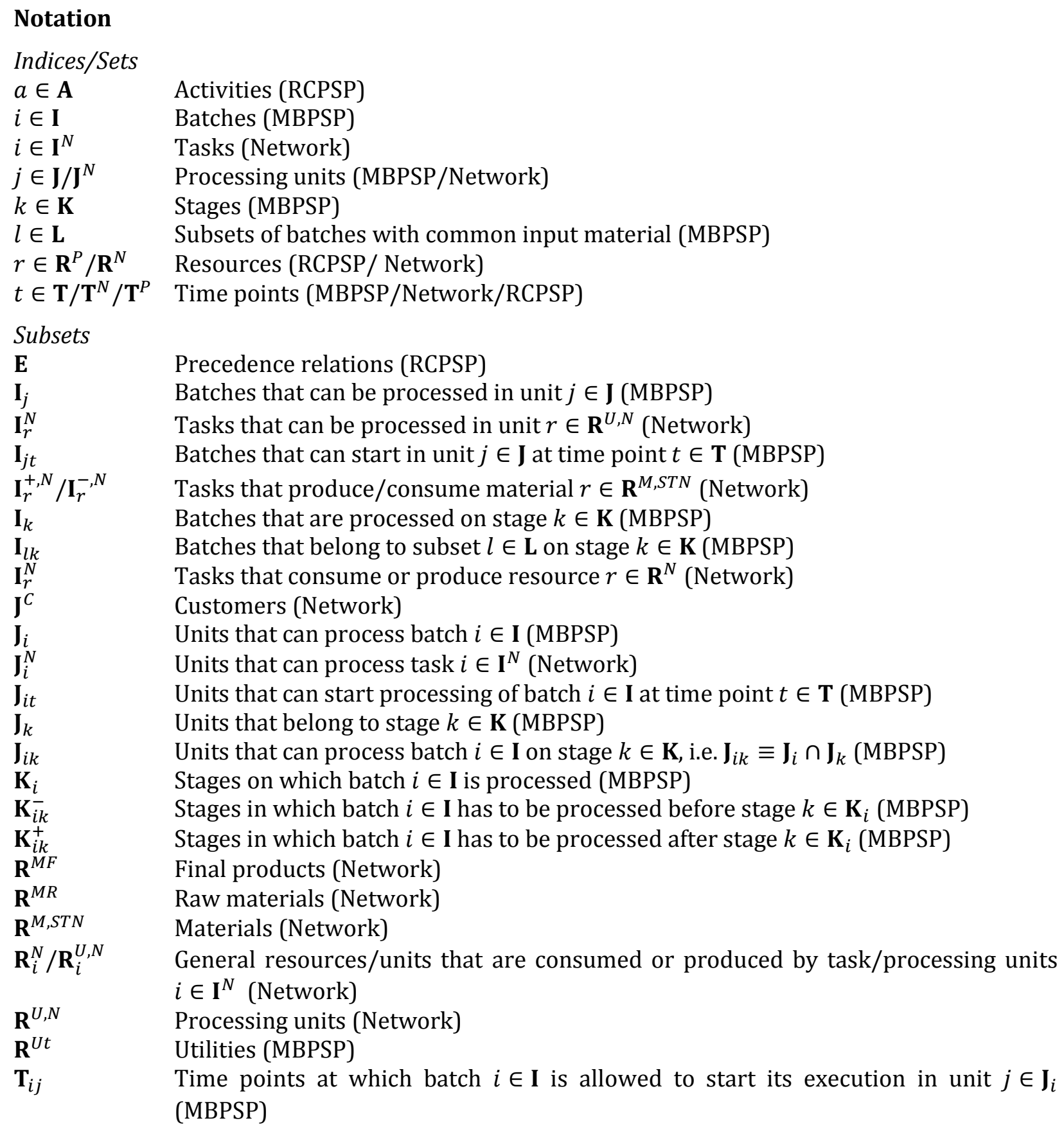

\section{Parameters}

$\alpha_{i j}$

$\alpha_{r t}^{U t}$

$\beta_{i r}^{m, N} / \beta_{i r}^{M, N}$

Fixed cost of processing batch $i \in \mathbf{I}$ in $j \in \mathbf{J}_{i}$ (MBPSP)

Cost of utility $r \in \mathbf{R}^{U t}$ during period $t \in \mathbf{T} \backslash\left\{\tau^{F}\right\}$ (MBPSP)

Minimum/maximum capacity utilization of resource $r \in \mathbf{R}^{N}$ by task $i \in \mathbf{I}_{r}^{N}$ (Network)

$\beta_{r}^{P} \quad$ Number of available units of resource $r \in \mathbf{R}$ (RCPSP)

$\beta_{a r}^{A, P}$

$\gamma_{k}$

$\delta$

Number of units of resource $r \in \mathbf{R}$ required by activity $a \in \mathbf{A}$ (RCPSP)

Lower bound on the sum of actual start times on stage $k \in \mathbf{K}$ (RCPSP)

Step size for time discretization (MBPSP)

$\varepsilon_{j}$

Earliest start time for unit $j \in \mathbf{J}$ (MBPSP) 


\begin{tabular}{|c|c|}
\hline$\varepsilon_{i j}^{U} / \varepsilon_{i k}^{S}$ & Earliest start time for batch $i \in \mathbf{I}$ in unit $j \in \mathbf{J}_{i} /$ on stage $k \in \mathbf{K}_{i}$ (MBPSP) \\
\hline$\zeta_{k}$ & Lower bound on the sum of actual tails on stage $k \in \mathbf{K}$ (RCPSP) \\
\hline$\eta^{N}$ & Scheduling horizon (Network) \\
\hline$\eta^{P}$ & Project duration (RCPSP) \\
\hline$\theta_{j}$ & Time window for unit $j \in \mathbf{J}$ (MBPSP) \\
\hline$\theta_{i j}^{U, N}$ & Available time window for task $i \in \mathbf{I}^{N}$ in unit $j \in \mathbf{J}_{i}^{N}$ (Network) \\
\hline$\kappa_{i}^{F S} / \kappa_{i}^{L S}$ & First/last stage on which batch $i \in \mathbf{I}$ can be processed (MBPSP) \\
\hline$\kappa_{j}$ & Stage to which unit $j \in \mathrm{J}$ belongs (MBPSP) \\
\hline$\lambda_{j}$ & Latest finish time for unit $j \in \mathbf{J}$ (MBPSP) \\
\hline$\lambda_{i j}^{U} / \lambda_{i k}^{S}$ & Latest finish time for batch $i \in \mathbf{I}$ in unit $j \in \mathbf{J}_{i} /$ on stage $k \in \mathbf{K}_{i}$ (MBPSP) \\
\hline $\bar{\mu}_{i} / \mu_{i}$ & Release time for batch $i \in \mathbf{I}$ in real time/in terms of time grid (MBPSP) \\
\hline$v_{i k}$ & $\begin{array}{l}\text { Defines the next stage on which batch } i \text { can be processed after stage } k \in \mathbf{K}_{i} \text {, } \\
\text { (MBPSP) }\end{array}$ \\
\hline$\xi_{r t}^{N}$ & Amount of resource $r \in \mathbf{R}^{N}$ made available or unavailable at time $t \in \mathbf{T}^{N}$ (Network) \\
\hline$\rho_{i r t}^{N}$ & $\begin{array}{l}\text { Number of variable units of resource } r \in \mathbf{R}^{N} \text { that are required by task } i \in \mathbf{I}_{r}^{N} \text { at } t \\
\text { time points after the beginning of the task }\end{array}$ \\
\hline$\rho_{i r}^{S T N}$ & $\begin{array}{l}\text { Conversion coefficient for material } r \in \mathbf{R}^{M, S T N} \text { produced or consumed by task } i \in \mathbf{I}^{N} \\
\text { (Network) }\end{array}$ \\
\hline$\sigma_{j}$ & Shortest tail of unit $j \in \mathbf{J}$ (MBPSP) \\
\hline$\sigma_{i j}^{U, N}$ & Shortest tail of task $i \in \mathbf{I}^{N}$ in unit $j \in \mathbf{J}_{i}^{N}$ (Network) \\
\hline$\sigma_{i k}^{S}$ & Shortest tail of batch $i \in \mathbf{I}$ on stage $k \in \mathbf{K}_{i}$ (MBPSP) \\
\hline$\tau^{0} / \tau^{F}$ & Start/end of the scheduling horizon (MBPSP) \\
\hline$\tau_{a}^{P}$ & Duration of activity $a \in \mathbf{A}$ (RCPSP) \\
\hline $\begin{array}{l}\bar{\tau}_{i j} / \tau_{i j} \\
\text { (MBPSP) }\end{array}$ & Processing time of batch $i \in \mathbf{I}$ in unit $j \in \mathbf{J}$ in real time/in terms of time grid \\
\hline $\bar{\tau}_{i}^{N} / \tau_{i}^{N}$ & Duration of task $i \in \mathbf{I}^{N}$ in real time units/in terms of time grid (Network) \\
\hline $\bar{\phi}_{i} / \phi_{i}$ & Due date for batch $i \in \mathbf{I}$ in real time/in terms of time grid (MBPSP) \\
\hline $\bar{\phi}_{r j}^{C} / \phi_{j r}^{C}$ & $\begin{array}{l}\text { Due date of product } r \in \mathbf{R}^{M F} \text { for customer } j \in \mathbf{J}^{C} \text { in real time/in terms of time grid } \\
\text { (Network) }\end{array}$ \\
\hline $\bar{\phi}_{r}^{N} / \phi_{r}^{N}$ & Due date for final product $R \in \mathbf{R}^{M F}$ in real time/in terms of time grid (Network) \\
\hline$\chi_{i j r}$ & Fixed requirement of utility $r \in \mathbf{R}^{U t}$ for batch $i \in \mathbf{I}$ in unit $j \in \mathbf{J}_{i}$ (MBPSP) \\
\hline$\chi_{\text {irt }}^{R T N}$ & $\begin{array}{l}\text { Number of discrete units of resource } r \in \mathbf{R}^{R} \text { required by task } i \in \mathbf{I}_{r}^{R} \text { at } t \text { time points } \\
\text { after the beginning of the task (Network) }\end{array}$ \\
\hline$\psi_{r t}$ & Maximum availability/inventory of resource $r \in \mathbf{R}$ during period $t \in \mathbf{T} \backslash\{0\}$ (MBPSP) \\
\hline$\omega_{i i^{\prime} j k}^{\#}$ & Indicator parameters to identify redundant constraints when defining model \\
\hline & MH\&G_LP used to refine discrete-time schedules, \# $\in\{1,2,3\}$ (MBPSP) \\
\hline$M$ & Large number for continuous-time big-M formulations (RCPSP) \\
\hline \multicolumn{2}{|c|}{ Binary/integer variables } \\
\hline$N_{i r}^{N}$ & Number of batches of task $i \in \mathbf{I}^{N}$ in unit $r \in \mathbf{R}_{i}^{U, N}$ (Network) \\
\hline$N_{j}$ & Number of batches assigned to unit $j \in \mathbf{J}$ (MBPSP) \\
\hline$R_{j t}$ & One if unit $j \in \mathbf{J}$ is available during time period $t \in \mathbf{T} \backslash\left\{\tau^{F}\right\}$ (MBPSP) \\
\hline$R_{r t}^{\text {div }}$ & $\begin{array}{l}\text { Number of batches of material } r \in \mathbf{R}^{M, S T N} \text { is available during period } t \in \mathbf{T}^{N} \backslash\{0\} \\
\text { (Network) }\end{array}$ \\
\hline$S_{i k t}$ & $\begin{array}{l}\text { One if the material produced by batch } i \in \mathbf{I} \text { after stage } k \in \mathbf{K}_{i} \text { is available during } \\
\text { period } t \in \mathbf{T} \backslash\left\{\tau^{F}\right\} \text { (MBPSP) }\end{array}$ \\
\hline$X_{i j}^{C}$ & One if batch $i \in \mathbf{I}$ is assigned to unit $j \in \mathbf{J}_{i}$ (MBPSP, continuous-time) \\
\hline
\end{tabular}


$X_{i j t} \quad$ One if batch $i \in \mathbf{I}$ starts being processed in unit $j \in \mathbf{J}_{i}$ at time $t \in \mathbf{T}_{i j}$ (MBPSP)

$X_{i t}^{N} \quad$ One if task $i \in \mathbf{I}^{N}$ starts at time $t \in \mathbf{T}^{N}$ (Network)

$X_{i j t}^{N} / X_{i r t}^{N} \quad$ One if task $i \in \mathbf{I}^{N}$ starts in unit $j \in \mathbf{J}_{i}^{N} / r \in \mathbf{R}_{i}^{U, N}$ at time $t \in \mathbf{T}^{N}$ (Network)

$Y_{i i^{\prime} k} \quad$ One if batch $i \in \mathbf{I}$ is processed before batch $i^{\prime} \in \mathbf{I}$ on stage $k \in \mathbf{K}_{i} \cap \mathbf{K}_{i^{\prime}}$ (MBPSP, continuous-time)

$Z_{r j t}^{N} \quad$ One if product $r \in \mathbf{R}^{M F}$ is delivered to customer $j \in \mathbf{J}^{C}$ at time $t \in \mathbf{T}^{N}$ (Network)

Continuous variables

$B_{i t}^{N} \quad$ Extent of task $i \in \mathbf{I}_{r}^{N}$ during period $t \in \mathbf{T}^{N} \backslash\{0\}$ (Network)

$C_{i k} \quad$ Completion time for batch $i \in \mathbf{I}$ on stage $k \in \mathbf{K}_{i}$ (MBPSP, continuous-time)

$E_{i k} \quad$ Actual start time of batch $i \in \mathbf{I}$ on stage $k \in \mathbf{K}_{i}$ (MBPSP)

$E_{i j}^{N} \quad$ Actual start time of task $i \in \mathbf{I}^{N}$ in unit $j \in \mathbf{J}_{i}^{N}$ (Network)

$G_{k} \quad$ Busyness of stage $k \in \mathbf{K}$ (MBPSP)

$H \quad$ Variable end of the scheduling horizon (MBPSP)

MS Makespan (MBPSP, Network)

$R_{r t}^{N} \quad$ Excess resource/inventory of $r \in \mathbf{R}^{N}$ during period $t \in \mathbf{T}^{N} \backslash\{0\}$ (Network)

$T_{i k} \quad$ Actual tail of batch $i \in \mathbf{I}$ on stage $k \in \mathbf{K}_{i}$ (MBPSP)

$T_{i r}^{N} \quad$ Actual start time and tail of task $i \in \mathbf{I}^{N}$ in unit $r \in \mathbf{R}_{i}^{U, N}$ (Network)

$W_{r t} \quad$ Amount of utility $r \in \mathbf{R}^{U t}$ consumed during time period $t \in \mathbf{T} \backslash\left\{\tau^{F}\right\}$ (MBPSP)

Other symbols

$\mathbb{S}(\cdot)$

$\mathbb{T}(\cdot)$

Materials (states) in the STN representation

Tasks in the STN representation

$\mathbb{U}$.

Processing units in the RTN representation and the MPSPS description

\section{Acknowledgments}

The authors acknowledge financial support from the National Science Foundation under Grants CBET-1066206 and CBET-1264096, and from the American Chemical Society - Petroleum Research Fund under Grant PRF-53313ND9. 


\section{References}

Bassett, M. H., Pekny, J. F. and Reklaitis, G. V. (1996) Decomposition techniques for the solution of large-scale scheduling problems. AlChE J. 42, 3373-3387.

Blau, G. E., Pekny, J. F., Varma, V. A. and Bunch, P. R. (2004) Managing a portfolio of interdependent new product candidates in the pharmaceutical industry. Journal of Product Innovation Management. 21, 227-245.

Brah, S. A. (1988) Scheduling in a Flow Shop with Multiple Processors. PhD thesis, University of Houston.

Brucker, P., Drexl, A., Mohring, R., Neumann, K. and Pesch, E. (1999) Resource-constrained project scheduling: Notation, classification, models, and methods. European Journal of Operational Research. 112, 3-41.

Capon-Garcia, E., Ferrer-Nadal, S., Graells, M. and Puigjaner, L. (2009) An Extended Formulation for the Flexible Short-Term Scheduling of Multiproduct Semicontinuous Plants. Industrial \& Engineering Chemistry Research. 48, 2009-2019.

Castro, P. M. and Grossmann, I. E. (2005) New continuous-time MILP model for the short-term scheduling of multistage batch plants. Ind. Eng. Chem. Res. 44, 9175-9190.

Castro, P. M. and Grossmann, I. E. (2006) An efficient MILP model for the short-term scheduling of single stage batch plants. Computers \& Chemical Engineering. 30, 1003-1018.

Castro, P. M., Hariunkoski, I. and Grossmann, I. E. (2009) Optimal Short-Term Scheduling of LargeScale Multistage Batch Plants. Ind. Eng. Chem. Res. 48, 11002-11016.

Chen, C. L., Liu, C. L., Feng, X. D. and Shao, H. H. (2002) Optimal short-term scheduling of multiproduct single-stage batch plants with parallel lines. Ind. Eng. Chem. Res. 41, 12491260.

Christofides, N., Alvarez-Valdes, R. and Tamarit, J. M. (1987) Project Scheduling with Resource Constraints - A Branch and Bound Approach. Eur J Oper Res. 29, 262-273.

Colvin, M. and Maravelias, C. T. (2010) Modeling methods and a branch and cut algorithm for pharmaceutical clinical trial planning using stochastic programming. Eur J Oper Res. 203, 205-215.

Colvin, M. and Maravelias, C. T. (2011) R\&D pipeline management: Task interdependencies and risk management. European Journal of Operational Research. 215, 616-628.

Demeulemeester, E. L. and Herroelen, W. (2002) Project scheduling: a research handbook. Springer Science \& Business Media

Dolan, E. D. and More, J. J. (2002) Benchmarking optimization software with performance profiles. Mathematical Programming. 91, 201-213.

Egli, U. M. and Rippin, D. W. T. (1986) Short-Term Scheduling for Multiproduct Batch ChemicalPlants. Computers \& Chemical Engineering. 10, 303-325.

Floudas, C. A. and Lin, X. X. (2004) Continuous-time versus discrete-time approaches for scheduling of chemical processes: a review. Comput Chem Eng. 28, 2109-2129.

Graves, S. C. (1981) A Review of Production Scheduling. Oper. Res. 29, 646-675.

Gupta, S. and Karimi, I. A. (2003) An improved MILP formulation for scheduling multiproduct, multistage batch plants. Ind. Eng. Chem. Res. 42, 2365-2380. 
Harjunkoski, I. and Grossmann, I. E. (2002) Decomposition techniques for multistage scheduling problems using mixed-integer and constraint programming methods. Comput Chem Eng. 26, 1533-1552.

Harjunkoski, I., Maravelias, C. T., Bongers, P., Castro, P. M., Engell, S., Grossmann, I. E., Hooker, J., Mendez, C., Sand, G. and Wassick, J. (2014) Scope for industrial applications of production scheduling models and solution methods. Computers \& Chemical Engineering. 62, 161-193.

Hartmann, S. and Briskorn, D. (2010) A survey of variants and extensions of the resourceconstrained project scheduling problem. Eur J Oper Res. 207, 1-14.

Jain, V. and Grossmann, I. E. (1999) Resource-constrained scheduling of tests in new product development. Industrial \& Engineering Chemistry Research. 38, 3013-3026.

Kallrath, J. (2002) Planning and scheduling in the process industry. Or Spectrum. 24, 219-250.

Kelly, J. D. and Zyngier, D. (2008) Hierarchical decomposition heuristic for scheduling: Coordinated reasoning for decentralized and distributed decision-making problems. Comput Chem Eng. 32, 2684-2705.

Kelly, J. D. and Zyngier, D. (2009) Multi-product inventory logistics modeling in the process industries Optimization and logistics challenges in the enterprise (Chaovalitwongse, W., Furman, K. C. and Pardalos, P. M. eds.), 61-95, Springer.

Kondili, E., Pantelides, C. C. and Sargent, R. W. H. (1993) A General Algorithm for Short-Term Scheduling of Batch-Operations .1. Milp Formulation. Comput Chem Eng. 17, 211-227.

Kondili, E., Pantelides, C. C. and Sargent, R. W. H. (1993) A General Algorithm for Short-Term Scheduling of Batch-Operations - I. MILP Formulation. Computers \& Chemical Engineering. 17, 211-227.

Kopanos, G. M., Kyriakidis, T. S. and Georgiadis, M. C. (2014) New continuous-time and discretetime mathematical formulations for resource-constrained project scheduling problems. Comput Chem Eng. 68, 96-106.

Kopanos, G. M., Miguel Lainez, J. and Puigjaner, L. (2009) An Efficient Mixed-Integer Linear Programming Scheduling Framework for Addressing Sequence-Dependent Setup Issues in Batch Plants. Industrial \& Engineering Chemistry Research. 48, 6346-6357.

Kyriakidis, T. S., Kopanos, G. M. and Georgiadis, M. C. (2012) MILP formulations for single- and multi-mode resource-constrained project scheduling problems. Comput Chem Eng. 36, 369385.

Linn, R. and Zhang, W. (1999) Hybrid flow shop scheduling: A survey. Computers \& Industrial Engineering. 37, 57-61.

Liu, Y. and Karimi, I. A. (2007) Novel continuous-time formulations for scheduling multi-stage batch plants with identical parallel units. Computers \& Chemical Engineering. 31, 1671-1693.

Liu, Y. and Karimi, I. A. (2007) Scheduling multistage, multiproduct batch plants with nonidentical parallel units and unlimited intermediate storage. Chem Eng Sci. 62, 1549-1566.

Maccarthy, B. L. and Liu, J. Y. (1993) Addressing the Gap in Scheduling Research - A Review of Optimization and Heuristic Methods in Production Scheduling. International Journal of Production Research. 31, 59-79.

Maravelias, C. T. (2005) Mixed-time representation for state-task network models. Ind. Eng. Chem. Res. 44, 9129-9145. 
Maravelias, C. T. (2006) A decomposition framework for the scheduling of single- and multi-stage processes. Comput Chem Eng. 30, 407-420.

Maravelias, C. T. (2012) General framework and modeling approach classification for chemical production scheduling. Aiche Journal. 58, 1812-1828.

Maravelias, C. T. and Grossmann, I. E. (2003) Minimization of the makespan with a discrete-time state-task network formulation. Ind. Eng. Chem. Res. 42, 6252-6257.

Maravelias, C. T. and Grossmann, I. E. (2004) A hybrid MILP/CP decomposition approach for the continuous time scheduling of multipurpose batch plants. Comput Chem Eng. 28, 19211949.

Mauderli, A. and Rippin, D. W. T. (1979) Production Planning and Scheduling for Multipurpose Batch Chemical Plants. Computers \& Chemical Engineering. 3, 199-206.

Mendez, C. A., Cerda, J., Grossmann, I. E., Harjunkoski, I. and Fahl, M. (2006) State-of-the-art review of optimization methods for short-term scheduling of batch processes. Comput Chem Eng. 30, 913-946.

Mendez, C. A., Henning, G. P. and Cerda, J. (2000) Optimal scheduling of batch plants satisfying multiple product orders with different due-dates. Comput Chem Eng. 24, 2223-2245.

Mendez, C. A., Henning, G. P. and Cerda, J. (2001) An MILP continuous-time approach to short-term scheduling of resource-constrained multistage flowshop batch facilities. Comput Chem Eng. 25, 701-711.

Merchan, A. F. and Maravelias, C. T. (2014) Reformulations of Mixed-Integer Programming Continuous-Time Models for Chemical Production Scheduling. Ind. Eng. Chem. Res. 53, 10155-10165.

Merchan, A. F. and Maravelias, C. T. (2016) Preprocessing and tightening methods for time-indexed MIP chemical production scheduling models. Computers \& Chemical Engineering. 84, 516535.

Merchan, A. F., Velez, S. and Maravelias, C. T. (2013) Tightening methods for continuous-time mixed-integer programming models for chemical production scheduling. AlChE J. 59, 44614467.

Pantelides, C. C. (1994) Unified frameworks for optimal process planning and scheduling. Proceedings on the second conference on foundations of computer aided operations. 253-274.

Papageorgiou, L. G., Rotstein, G. E. and Shah, N. (2001) Strategic supply chain optimization for the pharmaceutical industries. Ind. Eng. Chem. Res. 40, 275-286.

Pinedo, M. (2005) Planning and scheduling in manufacturing and services. Springer

Pinedo, M. L. (2012) Scheduling: theory, algorithms, and systems. Springer Science \& Business Media

Pinto, J. M. and Grossmann, I. E. (1995) A Continuous-Time Mixed-Integer Linear-Programming Model for Short-Term Scheduling of Multistage Batch Plants. Ind. Eng. Chem. Res. 34, 30373051.

Pinto, J. M. and Grossmann, I. E. (1998) Assignment and sequencing models for the scheduling of process systems. Annals of Operations Research. 81, 433-466.

Pritsker, A. A. B., Waiters, L. J. and Wolfe, P. M. (1969) Multiproject Scheduling with Limited Resources: A Zero-One Programming Approach. Management Science. 16, 93-108. 
Reklaitis, G. V. (1996) Overview of scheduling and planning of batch process operations. Batch processing systems engineering, 660-705, Springer.

Roe, B., Papageorgiou, L. G. and Shah, N. (2005) A hybrid MILP/CLP algorithm for multipurpose batch process scheduling. Comput Chem Eng. 29, 1277-1291.

Ruiz, R. and Vazquez-Rodriguez, J. A. (2010) The hybrid flow shop scheduling problem. Eur J Oper Res. 205, 1-18.

Schilling, G. and Pantelides, C. C. (1996) A simple continuous-time process scheduling formulation and a novel solution algorithm. Comput Chem Eng. 20, S1221-S1226.

Schmidt, C. W. and Grossmann, I. E. (1996) Optimization models for the scheduling of testing tasks in new product development. Industrial \& Engineering Chemistry Research. 35, 3498-3510.

Shah, N. (1998) Single and Multisite Planning and Scheduling: Current Status and Future Challenges. AIChE Symposium Series. 75-90.

Shah, N., Pantelides, C. C. and Sargent, R. W. H. (1993) A General Algorithm for Short-Term Scheduling of Batch-Operations - II. Computational Issues. Computers \& Chemical Engineering. 17, 229-244.

Subrahmanyam, S., Kudva, G. K., Bassett, H. H. and Pekny, J. F. (1996) Application of distributed computing to batch plant design and scheduling. AlChE J. 42, 1648-1661.

Subramanian, D., Pekny, J. F. and Reklaitis, G. V. (2001) A simulation-optimization framework for Research and Development Pipeline management. AlChE J. 47, 2226-2242.

Sundaramoorthy, A. and Karimi, I. A. (2005) A simpler better slot-based continuous-time formulation for short-term scheduling in multipurpose batch plants. Chem Eng Sci. 60, 2679-2702.

Sundaramoorthy, A. and Maravelias, C. T. (2011) A General Framework for Process Scheduling. Aiche Journal. 57, 695-710.

Sundaramoorthy, A. and Maravelias, C. T. (2011) Computational Study of Network-Based MixedInteger Programming Approaches for Chemical Production Scheduling. Industrial \& Engineering Chemistry Research. 50, 5023-5040.

Sundaramoorthy, A., Maravelias, C. T. and Prasad, P. (2009) Scheduling of Multistage Batch Processes under Utility Constraints. Ind. Eng. Chem. Res. 48, 6050-6058.

Talbot, F. B. (1982) Resource-Constrained Project Scheduling with Time-Resource Tradeoffs - The Non-Preemptive Case. Manage Sci. 28, 1197-1210.

Velez, S. and Maravelias, C. T. (2013) A branch-and-bound algorithm for the solution of chemical production scheduling MIP models using parallel computing. Comput Chem Eng. 55, 28-39.

Velez, S. and Maravelias, C. T. (2013) Mixed-Integer Programming Model and Tightening Methods for Scheduling in General Chemical Production Environments. Ind. Eng. Chem. Res. 52, 34073423.

Velez, S. and Maravelias, C. T. (2013) Multiple and nonuniform time grids in discrete-time MIP models for chemical production scheduling. Comput Chem Eng. 53, 70-85.

Velez, S. and Maravelias, C. T. (2013) Reformulations and Branching Methods for Mixed-Integer Programming Chemical Production Scheduling Models. Ind. Eng. Chem. Res. 52, 3832-3841. 
Velez, S. and Maravelias, C. T. (2014) Advances in mixed-integer programming methods for chemical production scheduling. Annu Rev Chem Biomol Eng. 5, 97-121.

Velez, S. and Maravelias, C. T. (2015) Theoretical framework for formulating MIP scheduling models with multiple and non-uniform discrete-time grids. Comput Chem Eng. 72, 233-254.

Velez, S., Merchan, A. F. and Maravelias, C. T. (2015) On the solution of large-scale mixed integer programming scheduling models. Chemical Engineering Science. 136, 139-157.

Velez, S., Sundaramoorthy, A. and Maravelias, C. T. (2013) Valid Inequalities Based on Demand Propagation for Chemical Production Scheduling MIP Models. AlChE J. 59, 872-887.

Wang, H. (2005) Flexible flow shop scheduling: optimum, heuristics and artificial intelligence solutions. Expert Systems. 22, 78-85.

Weglarz, J., Jozefowska, J., Mika, M. and Waligora, G. (2011) Project scheduling with finite or infinite number of activity processing modes - A survey. Eur J Oper Res. 208, 177-205.

Wiers, V. C. S. (1997) A review of the applicability of OR and AI scheduling techniques in practice. Omega-International Journal of Management Science. 25, 145-153.

Yee, K. L. and Shah, N. (1998) Improving the efficiency of discrete time scheduling formulation. Comput Chem Eng. 22, Supplement 1, S403-S410.

Zapata, J. C., Hodge, B. M. and Reklaitis, G. V. (2008) The multimode resource constrained multiproject scheduling problem: Alternative formulations. AlChE J. 54, 2101-2119. 